\title{
Identification of Transmembrane Domain 5 as a Critical Molecular Determinant of Menthol Sensitivity in Mammalian TRPA1 Channels
}

\author{
Bailong Xiao, ${ }^{1}$ Adrienne E. Dubin, ${ }^{1}$ Badry Bursulaya, ${ }^{2}$ Veena Viswanath, ${ }^{1}$ Timothy J. Jegla, ${ }^{1}$ and Ardem Patapoutian ${ }^{1,2}$ \\ ${ }^{1}$ Department of Cell Biology, The Scripps Research Institute, La Jolla, California 92037, and ${ }^{2}$ Genomics Institute of the Novartis Research Foundation, San \\ Diego, California 92121
}

\begin{abstract}
TRPA1 is a member of the transient receptor potential (TRP) family of ion channels and is expressed in a subset of nociceptive neurons. An increasing body of evidence suggests that TRPA1 functions as a chemical nocisensor for a variety of reactive chemicals, such as pungent natural compounds and environmental irritants. Activation of TRPA1 by reactive compounds has been demonstrated to be mediated through covalent modification of cytoplasmic cysteines located in the $\mathrm{N}$ terminus of the channel, rather than classical lockand-key binding. TRPA1 activity is also modulated by numerous nonreactive chemicals, but the underlying mechanism is unknown. Menthol, a natural nonreactive cooling compound, is best known as an activator of TRPM8, a related TRP ion channel required for cool thermosensation in vivo. More recently, menthol has been shown to be an activator of mouse TRPA1 at low concentrations, and a blocker, at high concentrations. Here, we show that human TRPA1 is only activated by menthol, whereas TRPA1 from nonmammalian species are insensitive to menthol. Mouse-human TRPA1 chimeras reveal the pore region [including transmembrane domain 5 (TM5) and TM6] as the critical domain determining whether menthol can act as an inhibitor. Furthermore, chimeras between Drosophila melanogaster and mammalian TRPA1 highlight specific residues within TM5 critical for menthol responsiveness. Interestingly, this TM5 region also determines the sensitivity of TRPA1 to other chemical modulators. These data suggest separable structural requirements for modulation of TRPA1 by covalent and nonreactive molecules. Whether this region is involved in binding or gating of TRPA1 channels is discussed.
\end{abstract}

Key words: transient receptor potential; TRPA1; menthol; chemical nocisensor; nociception; binding pocket

\section{Introduction}

TRPA1 is a member of the transient receptor potential (TRP) family of nonselective cation channels, many of which are involved in sensing diverse chemical and physical stimuli (Clapham, 2003; Voets et al., 2005; Dhaka et al., 2006). In mammals, TRPA1 is expressed in nociceptors and functions as an important component of the pain transduction machinery (Story et al., 2003; Jordt et al., 2004). TRPA1 is activated by pungent compounds (Bandell et al., 2004; Jordt et al., 2004; Macpherson et al., 2005; Kwan et al., 2006), noxious cold (Story et al., 2003; Sawada et al., 2007), hyperosmotic stress (Zhang et al., 2008), and intracellular $\mathrm{Ca}^{2+}$ (Doerner et al., 2007; Zurborg et al., 2007). Although the role of TRPA1 in sensing noxious cold and somatic mechanosensation in vivo remains unsettled (Obata et al., 2005; Bautista et al., 2006; Kwan et al., 2006; Petrus et al., 2007), a role for TRPA1 is established as a chemical nocisensor for a wide

Received June 16, 2008; revised Aug. 5, 2008; accepted Aug. 12, 2008.

This work was supported by National Institutes of Health Grants DE016927 and NS046303 and by the Novartis Research Foundation. B.X. is the recipient of a Canadian Institutes of Health Research Fellowship Award and Alberta Heritage Foundation for Medical Research Fellowship Award. We thank Dr. Jorg Grandl for critical reading of this manuscript and helpful discussion.

Correspondence should be addressed to Ardem Patapoutian, Department of Cell Biology, ICND202, The Scripps Research Institute, 10550 North Torrey Pines Road, La Jolla, CA 92037. E-mail: apatapou@gnf.org. DOI:10.1523/JNEUROSCI.2772-08.2008

Copyright $\odot 2008$ Society for Neuroscience $\quad$ 0270-6474/08/289640-12\$15.00/0 variety of reactive compounds, including pungent natural substances such as mustard oil (MO), cinnamaldehyde, and allicin, environmental irritants such as acrolein, endogenous lipid products such as 4-hydroxynonenal, and formalin (Bautista et al., 2006; Kwan et al., 2006; Macpherson et al., 2007b; McNamara et al., 2007; Trevisani et al., 2007; Andersson et al., 2008). In addition to these reactive chemicals, numerous nonreactive organic chemicals have also been shown to activate TRPA1, such as icilin (Story et al., 2003), trinitrophenol (Hill and Schaefer, 2007), farnesyl thiosalicylic acid (FTS) (Maher et al., 2008), and clotrimazole (Meseguer et al., 2008).

To function as a broad polymodal nocisensor, it is conceivable that TRPA1 has used multiple mechanisms for sensing chemically diverse compounds signaling a broad repertoire of chemicals. Recently, two independent research groups have demonstrated that reactive compounds activate TRPA1 by covalently modifying cysteine residues located in the $\mathrm{N}$ terminus of the channel, rather than a classical lock-and-key binding mechanism (Hinman et al., 2006; Macpherson et al., 2007a). Mutating specific cytoplasmic cysteine residues markedly impaired the sensitivity of TRPA 1 to those reactive compounds. In contrast, the mechanism underlying the modification of TRPA1 by nonreactive organic chemicals is unknown.

Menthol, a nonreactive compound derived from mint, is known to elicit a cool sensation. It is generally believed that men- 
thol sensation by mammalian sensory neurons is mediated by TRPM8 (McKemy et al., 2002; Peier et al., 2002), another member of cold-activated TRP channels required for cool thermosensation in vivo (Bautista et al., 2007; Colburn et al., 2007; Dhaka et al., 2007). However, many of these sensory chemicals have been shown to be promiscuous, affecting more than one thermoTRP. For example, we originally reported the inhibition of mouse TRPA1 (mTRPA1) activity by high concentrations of menthol (Macpherson et al., 2006). Interestingly, Karashima et al. (2007) have since shown that menthol has a bimodal action on mTRPA1. They confirmed the inhibitory action of menthol at high concentrations and importantly found that low concentrations resulted in channel activation. Here, we explore the molecular determinants of TRPA1 sensitivity to menthol.

\section{Materials and Methods}

Molecular cloning and mutagenesis. mTRPA1 full-length cDNA was cloned in the pcDNA5/FRT vector and in the IRES-yellow fluorescence protein (YFP) expression vector. human TRPA1 (hTRPA1) and Drosophila melanogaster TRPA1 (dTRPA1) full-length cDNA were cloned in pcDNA5/FRT vector, whereas TRPA1 from Takifugu (Fugu-TRPA1) and Anopheles gambiae (mosquito-TRPA1) were cloned in pcDNA3.1 vector. All chimeras were constructed using Stratagene QuickChange XL site-directed mutagenesis kit according to the manufacturer's instructions. Constructs were sequenced to confirm mutations and absence of errors. Amino acid compositions of important chimeric constructs are listed in supplemental Table 1. Other chimeras are depicted in supplemental Figure 1 (available at www.jneurosci.org as supplemental material).

Transient expression. Mammalian cell lines were transfected with mTRPA1-IRES-YFP or cotransfected with hTRPA1 or chimeras together with the YFP-expressing vector construct using FuGene 6 transfection reagent (Roche Diagnostics) according to the manufacturer's instructions. Chinese hamster ovary $(\mathrm{CHO})$ and human embryonic kidney 293T (HEK293T) cells were used for ratiometric calcium imaging and electrophysiological experiments, respectively. The choice of CHO cells for calcium imaging and HEK293T cells for electrophysiological experiments was based solely on the relative ease of single cell calcium imaging and patch-clamp recording from these cells, respectively. The conclusions drawn from data obtained from these cell lines using different experimental techniques were similar. For fluorometric imaging plate reader (FLIPR) studies, channel constructs were transfected in the absence of YFP.

Ratiometric $\mathrm{Ca}^{2+}$ imaging and FLIPR. Ratiometric $\mathrm{Ca}^{2+}$ imaging and FLIPR experiments were performed essentially as previously described (Macpherson et al., 2006). All calcium imaging graphs are averaged ratios of 20-80 individual cells. FLIPR experiments were used for $\mathrm{EC}_{50}$ and $\mathrm{IC}_{50}$ determinations. For the determination of menthol $\mathrm{IC}_{50}, 100 \mu \mathrm{M} \mathrm{MO}$ was coapplied with a range of menthol concentrations to the cells. For determination $\mathrm{IC}_{50}$ values for $(Z)$-4-(4-chlorophynyl)-3-methylbut-3en-2-oxime (AP18) and 4-methoxy- $N$-(2,2,2-trichloro-1-((4-chlorophenyl)sulfanyl)ethyl)benzamide (AMG5445), cells were exposed to a range of concentrations of either AP18 or AMG5445 for 3 min before the application of $30 \mu \mathrm{M} \mathrm{MO}$. Each point shown in the $\mathrm{EC}_{50}$ and $\mathrm{IC}_{50}$ curves is the average response of three or four wells and normalized to the response evoked by $100 \mu \mathrm{M}$ MO.

Electrophysiology. Whole-cell patch-clamp recordings were performed as described previously by Bandell et al. (2006) and Macpherson et al. (2007a) with the following modifications. Transfected HEK293T cells used for electrophysiological studies were usually cultured in the presence of $10 \mu \mathrm{M}$ ruthenium red and maintained for $12-18 \mathrm{~h}$ at $37^{\circ} \mathrm{C}$ followed by further incubation at $33^{\circ} \mathrm{C}$ for $1-3 \mathrm{~d}$ in $5 \% \mathrm{CO}_{2}$ before testing. Control coverslips were treated similarly. In most experiments studying menthol block of TRPA1 activation, a $\mathrm{Ca}^{2+}$ containing extracellular saline (ES) was used ( $2 \mathrm{~mm} \mathrm{Ca}^{2+} \mathrm{ES}$ ), which contained (in mM) 136 $\mathrm{NaCl}, 5 \mathrm{KCl}, 2 \mathrm{MgCl}_{2}, 2 \mathrm{CaCl}_{2}$, and 10 HEPES, pH 7.4 with $\mathrm{NaOH}$. In experiments studying the activation of TRPA1, the pipette solution [0
mM $\mathrm{Ca}^{2+}$ intracellular saline (IS)] contained (in mM) $124.5 \mathrm{CsCl}, 5$ EGTA, and 10 HEPES, pH 7.3 with $\mathrm{CsOH}$. In some experiments, the pipette contained $100 \mathrm{~nm}$ free $\mathrm{Ca}^{2+}$ buffered by BAPTA ( $1 \mathrm{~mm}$ ) (in mM): 130 methanesulfonate, $1 \mathrm{MgCl}_{2}, 0.483 \mathrm{CaCl}_{2}, 1 \mathrm{BAPTA}-\mathrm{K}$ salt, and 10 hemi-Na-HEPES, pH 7.3 with $\mathrm{CsOH}$.

TRPA1 activity was usually monitored using repetitive voltage ramps (at $5 \mathrm{~s}$ intervals) in the whole-cell configuration. Cells were held at -50 $\mathrm{mV}$, stepped to $-120 \mathrm{mV}$ briefly before a $2.8 \mathrm{mV} / \mathrm{ms}$ ramp to $+120 \mathrm{mV}$ and then held at $+120 \mathrm{mV}$ for $9 \mathrm{~ms}$ before returning to the holding potential. Using this protocol, small voltage-activated TRPA1dependent currents are observed above approximately $+80 \mathrm{mV}$ (see Fig. $2 A-C$, left, black traces) consistent with the reported voltage dependence of TRPA1. To quantify the effects of TRPA1 modulators on TRPA1 activity, averaged inward currents at $-120 \mathrm{mV}$ and averaged (over the first $\sim 5 \mathrm{~ms}$ ) outward currents at $+120 \mathrm{mV}$ were measured (see Fig. $2 \mathrm{~A}, \mathrm{C}$, middle). For statistical analysis, the currents were normalized to cell capacitance. In all voltage-ramp protocols a short step to $-75 \mathrm{mV}$ was performed at the beginning of each sweep to monitor membrane resistance and assess the stability of the access resistance (see Fig. $2 \mathrm{~B}$, left). Cells were continuously perfused with external saline and maintained at $25-27^{\circ} \mathrm{C}$ unless indicated. The time of compound arrival and initiation of washout was marked by the arrival of a small bubble between solutions. Cells in whole-cell configuration were challenged with a voltage step protocol to investigate the current-voltage relationship for TRPA1 activation by voltage. The series resistance compensation was $50 \%$. Tail currents were measured at $-120 \mathrm{mV}$ (supplemental Fig. $4 B$, inset, available at www.jneurosci.org as supplemental material). Noisy TRPA1 activity was observed on top of a smooth outward current with a bell shaped voltage dependence (data not shown). Before rupturing the patch to achieve a whole-cell recording, voltage protocols were applied to the cell-attached patch and voltage-dependent activation of TRPA1 channel activity was monitored (supplemental Fig. 4A,G,I, available at www. jneurosci.org as supplemental material).

Glutathione adduct formation. Compound reactivity was assayed as described previously (Macpherson et al., 2007a). Briefly, equimolar concentrations of TRPA1 modulators and glutathione (Sigma) were mixed in water and reacted at room temperature for $2 \mathrm{~h}$. Samples were then diluted in $\mathrm{MeOH}$ and subject to electrospray ionization mass spectrometry analysis.

Modeling and molecule docking. The mouse TRPA1 homology model was built using Prime 1.6 software from FirstDiscovery suite (Shelley et al., 2007) and KcsA (1BL8.pdb) (Doyle et al., 1998) as a template. The tetramer structure was embedded into a $70 \times 70 \AA$ bilayer composed of POPE (1-palmytoyl-2-olcoyl-sn-glycero-3-phosphatidylethanolamine) lipid molecules and solvated in a TIP3P water box in the presence of 0.5 $\mathrm{M} \mathrm{NaCl}$. The complex was subject to molecular dynamics simulation under periodic boundary conditions $(90 \times 90 \times 100 \AA)$. The simulation was initiated by minimizing the entire system in which protein backbone atoms were restrained with harmonic force of $10 \mathrm{kcal} / \mathrm{mol} \AA^{2}$. That was followed by 150 ps of constant volume and subsequent 1 ns of constantpressure molecular dynamics (MD) run at $310 \mathrm{~K}$. Bonds involving hydrogen atoms were constrained via the SHAKE algorithm. A $12 \AA$ cutoff distance was used for all nonbonded interactions. The time step for integration was $1 \mathrm{fs}$, and the coordinates of all atoms were saved every 5 ps. All simulations were performed using the NAMD software package (Phillips et al., 2005) with a charm27 force field. All calculations were performed on a cluster consisting of $2562.4 \mathrm{MHz}$ Opteron processors. Each MD simulation was run in parallel distributed over 16 processors and required on average 1 week to complete a $1 \mathrm{~ns}$ MD simulation run.

The optimized complex was used for docking calculations with Glide 2.6 (Shelley et al., 2007). The putative binding site of menthol and AP18 in the equilibrated complex was identified using SiteMap tool from FirstDiscovery suite (Shelley et al., 2007).

Reagents. (-)-Menthol, thymol, MO (allylisothiocyanate), trinitrophenol, ruthenium red, and AMG5445 were purchased from SigmaAldrich. FTS was purchased from Toronto Research Chemicals. AP18 was purchased from Maybridge or Biomol International. 2-(1,3dimethyl-2,6-dioxo-1,2,3,6-tetrahydro-7H-purin-7-yl)- $N$-(4-isopropylphenyl)acetamide (HC030031) was purchased from Chembridge. For 
preparing stock solutions, trinitrophenol and ruthenium red were dissolved in $\mathrm{H}_{2} \mathrm{O}$. ( - )-Menthol and thymol were dissolved in ethanol. MO, FTS, AMG5445, AP18, HC030031 were dissolved in DMSO. The chemical structures of (-)-menthol, thymol, FTS, AP18, AMG5445, and 4-methyl-N-[2,2,2-trichloro-1-(4-nitro-phenylsulfanyl)-ethyl]benzamide (CMP1) (Chen et al., 2008) are depicted in supplemental Figure 2 (available at www.jneurosci.org as supplemental material).

Data analysis. $\mathrm{EC}_{50}$ and $\mathrm{IC}_{50}$ values were determined by fitting data with a Hill Equation using IgorPro (Wavematrics). Data in all figures are shown as mean \pm SEM. Statistical significance was evaluated using unpaired Student's $t$ test.

\section{Results}

mTRPA1 and hTRPA1 respond differently to menthol

To determine the repertoire of functional effects of the cooling compound menthol on wild-type mTRPA1 and hTRPA1, the recombinant channels were expressed in $\mathrm{CHO}$ or HEK293T cells by transient transfection and their responsiveness to menthol and the TRPA1 agonist MO were assayed by ratiometric calcium imaging, FLIPR, and electrophysiology. All in vitro assay paradigms revealed that menthol modulated TRPA1 in a species-specific manner. In ratiometric calcium imaging, $250 \mu \mathrm{M}$ menthol induced an increase in intracellular $\mathrm{Ca}^{2+}$ in cells expressing hTRPA1, but not in those expressing mTRPA1 (Fig. 1A). However, subsequent rapid washout of menthol led to an increase in intracellular $\mathrm{Ca}^{2+}$ in cells expressing mTRPA1 that was not observed in cells expressing hTRPA1 (Fig. $1 A$ ). This "off-response" phenomenon of mTRPA1 has been described previously (Karashima et al., 2007). As reported previously (Macpherson et al., 2006), $250 \mu \mathrm{M}$ menthol blocked the response of mTRPA1 to $\mathrm{MO}$ (Fig. $1 \mathrm{~B}$ ). In contrast, there was no effect of $250 \mu \mathrm{M}$ menthol on the magnitude of the $\mathrm{MO}$-induced increase in $\mathrm{Ca}^{2+}$ in hTRPA1-expressing cells (Fig. $1 B$ ).

We next determined the menthol concentration-response of hTRPA1 and mTRPA1 using the FLIPR. Menthol activated hTRPA1 (Fig. 1C, Table 1). In addition, menthol did not block the response of hTRPA1 to $100 \mu \mathrm{M} \mathrm{MO}$ even at the highest concentration we tested ( $3 \mathrm{~mm}$ ) (Fig. 1D). These data indicate that menthol acts as an agonist of hTRPAl over a wide range of concentrations. In contrast, and similar to that reported by Karashima et al. (2007), mTRPA1 displayed a bell-shaped concentration-response curve to menthol (Fig. 1C). Whereas a concentration-dependent increase in intracellular $\mathrm{Ca}^{2+}$ levels in mTRPA1-transfected cells revealed activation at concentrations below100 $\mu \mathrm{M}$ menthol, $\mathrm{Ca}^{2+}$ levels declined in a concentrationdependent manner $>100 \mu \mathrm{M}$. The ability of menthol to inhibit responses to $100 \mu \mathrm{M} \mathrm{MO}$ was also determined (Fig. 1D). A concentration-dependent inhibition of the $\mathrm{MO}$ response at mTRPA1 was observed, reminiscent of the dependence observed during the declining phase of the bell-shaped agonist curve $\left(\mathrm{IC}_{50}\right.$ $=73.4 \pm 11.5 \mu \mathrm{M}$ ) (Fig. $1 \mathrm{D}$ ). The maximum activation of mTRPA1 by menthol was $<50 \%$ of that induced by $100 \mu \mathrm{M} \mathrm{MO}$, likely attributable to competing effects (Fig. $1 D$ ), so the efficacy of menthol activation of mTRPA1 is an underestimation. These data indicate that low concentrations of menthol activate mTRPA1, and that high concentrations inhibit the channel.

The responses of MTRPA1 and hTRPA1 to menthol were further characterized by whole-cell patch-clamp recordings from transiently transfected HEK293T cells. Consistent with the agonistic effect of menthol on hTRPA1 observed in calcium imaging and FLIPR, $250 \mu \mathrm{M}$ menthol evoked robust currents in cells expressing hTRPA1(Fig. $2 A)(59.0 \pm 10.2 \mathrm{pA} / \mathrm{pF}$ at $+120 \mathrm{mV} ; n=$ $11)$ but not control cells expressing YFP $(0.2 \pm 0.6 \mathrm{pA} / \mathrm{pF}$ at +120 $\mathrm{mV} ; n=6 ; p<0.0005)$. Voltage-ramp-induced currents in the
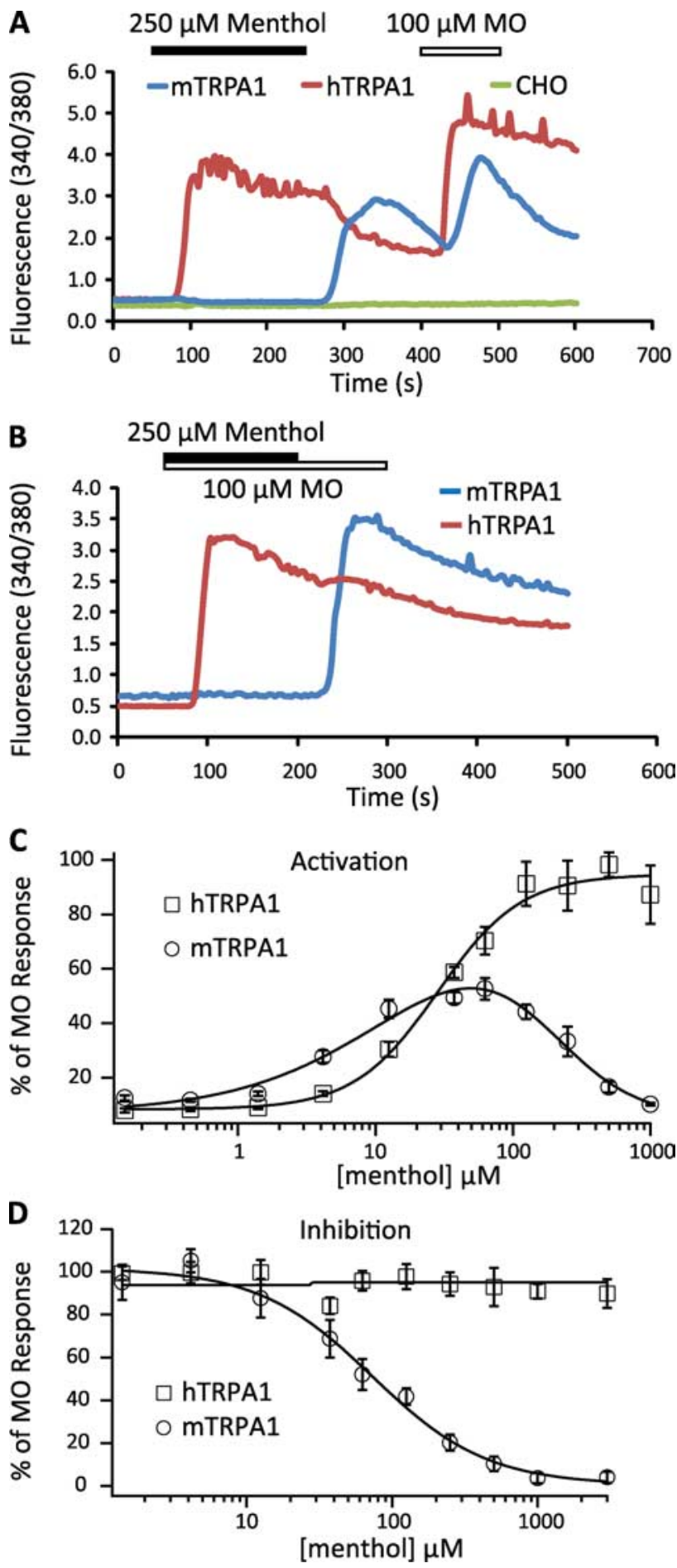

Figure 1. Distinct responses of hTRPA1 and MTRPA1 to menthol as assayed by ratiometric calcium imaging and FLIPR. $\boldsymbol{A}-\boldsymbol{D}$, The responsiveness of mTRPA1 and hTRPA1 to menthol was assayed by ratiometric calcium imaging $(\boldsymbol{A}, \boldsymbol{B})$ and $\operatorname{FLIPR}(\boldsymbol{C}, \boldsymbol{D})$. $\boldsymbol{A}$, Activation effect of menthol on $\mathrm{CHO}$ cells expressing hTRPA1 (red trace) and mTRPA1 (blue trace). Untransfected CHO cells responded neither to menthol nor to $\mathrm{MO}$ (green trace). Menthol $(250 \mu \mathrm{m})$ induced a robust increase in intracellular $\mathrm{Ca}^{2+}$ in cells expressing hTRPA1, but not in cells expressing MTRPA1. Notably, removal of menthol led to a rapid increase in intracellular $\mathrm{Ca}^{2+}$ in cells expressing mTRPA1. This phenomenon is termed "off response" and is a characteristic response of mTRPA1 to menthol activation. $\boldsymbol{B}$, Inhibitory effect of menthol on M0-evoked hTRPA1 (red trace) or mTRPA1 activity (blue trace). C, Menthol concentration-response relationships of hTRPA1 (open squares) or MTRPA1 (open circles). Note the bell-shaped sensitivity of mTRPA1 to menthol. $\boldsymbol{D}$, Concentration dependence of menthol to inhibit MO $(100 \mu \mathrm{m})$-evoked $\mathrm{Ca}^{2+}$ responses in HEK293T cells expressing hTRPA1 (open squares) or mTRPA1 (open circles). 
Table 1. Summary of the responsiveness of hTRPA1, hTRPA1-dTM5, and hTRPA1-S873V/T874L to TRPA1 modulators

\begin{tabular}{|c|c|c|c|c|}
\hline Compound & Effect & $\mathrm{hTRPA} \mathrm{EC}_{50}$ or IC $_{50}(\mu \mathrm{m})$ & hTRPA1-dTM5 $\mathrm{EC}_{50}$ or IC ${ }_{50}(\mu \mathrm{m})$ & hTRPA1-S873V/T874L EC 50 or IC $50(\mu \mathrm{m})$ \\
\hline Menthol & Activation & $28.4 \pm 3.6$ & No activation & $161.2 \pm 31.5$ \\
\hline Thymol & Activation & $62.5 \pm 3.2$ & No activation & $>400^{a}$ \\
\hline FTS & Activation & $7.3 \pm 1.1$ & No activation & $11.8 \pm 8.3$ \\
\hline Trinitrophenol & Activation & $383.8 \pm 5.7$ & $367.9 \pm 76.7$ & $218.6 \pm 3.6$ \\
\hline MO & Activation & $2.33 \pm 0.74$ & $1.32 \pm 0.46$ & $0.98 \pm 0.24$ \\
\hline AP18 & Inhibition & $0.60 \pm 0.17$ & No inhibition & No inhibition \\
\hline AMG5445 & Inhibition & $2.23 \pm 0.58$ & No inhibition & No inhibition \\
\hline HC030031 & Inhibition & $12.87 \pm 2.67$ & $11.47 \pm 2.39$ & $8.51 \pm 1.36$ \\
\hline Ruthenium red & Inhibition & $1.5 \pm 0.2$ & $0.48 \pm 0.03$ & $0.64 \pm 0.13$ \\
\hline
\end{tabular}

${ }^{a}$ Could not be accurately fit with a Hill Equation.

presence of menthol revealed outward rectification and deactivating tail currents (Fig. $2 \mathrm{~A}$, left, green trace) similar to currents activated by $100 \mu \mathrm{M} \mathrm{MO}$ (Fig. $2 A$, left, brown trace). The response to menthol was fully reversible after washout (Fig. $2 A$, right) and subsequent MO responses were similar to those observed in vehicle $(0.05 \%$ ethanol)-treated controls (data not shown). Menthol acted as an agonist whether $\mathrm{Ca}^{2+}$ was present or absent in internal and external recording salines suggesting that the agonistic effect of menthol on hTRPA1 is independent of $\mathrm{Ca}^{2+}$.

mTRPA1 was also strongly activated by $25-50 \mu \mathrm{M}$ menthol (Fig. $2 \mathrm{~B}$ ). Whole-cell current density at $+120 \mathrm{mV}$ was significantly greater in mTRPA1-transfected cells $(45.4 \pm 12.0 \mathrm{pA} / \mathrm{pF}$; $n=6)$ compared with vector-transfected YFP-positive control cells $(0.6 \pm 0.6 \mathrm{pA} / \mathrm{pF} ; n=4 ; p<0.02)$. Menthol induced outwardly rectifying currents that revealed slowly deactivating tail currents at $-120 \mathrm{mV}$ (Fig. $2 \mathrm{~B}$, left, green trace). Menthol caused a strong shift in voltage-dependent activation to more negative potentials readily observed in both the voltage step protocol and the prepulse voltage-dependent activation of tail currents similar to that reported by Karashima et al. (2007) (data not shown).

In agreement with the results obtained in calcium imaging studies, mTRPA1 differed from hTRPA1 when challenged with high concentrations of menthol. We independently corroborated the results reported by Karashima et al. (2007) that menthol at $500 \mu \mathrm{M}$ was a poorly efficacious agonist (Fig. 2 C). Only very small if any increases in voltage-ramp-induced currents were observed during challenge with $500 \mu \mathrm{M}$ menthol (Fig. $2 C$, top left, green trace; top right, current at $+120 \mathrm{mV}$ shortly after application of menthol). Subsequent application of MO together with menthol revealed only a small increase in conductance consistent with menthol-dependent block of MO sensitivity (Fig. 2C, top left, brown trace; top right, currents at $\pm 120 \mathrm{mV}$ under brown bar). Vehicle (0.05\% ethanol) had no effect on cellular conductance and $\mathrm{MO}$ was an effective agonist in its presence (Fig. $2 C$, bottom). Significant menthol-dependent inhibition of the MO response was observed using these protocols. Peak wholecell mTRPA1 currents at $+120 \mathrm{mV}$ elicited by $100 \mu \mathrm{M} \mathrm{MO}$ in the presence of $500 \mu \mathrm{M}$ menthol or vehicle were measured and normalized to the leak-subtracted current activated by step to $+180 \mathrm{mV}$ in each cell (as described by Macpherson et al., 2007a). MO-induced currents were significantly reduced in the presence of menthol compared with those in the presence of vehicle $[16 \pm 7 \%(n=7)$ vs $143 \pm 34 \%(n=4)$ of the currents elicited at $+180 \mathrm{mV}$, respectively; $p<0.05$ ]. Identical conclusions are drawn when $\mathrm{MO}$ responses are normalized to cell size.

The region spanning transmembrane domain 5 and transmembrane domain 6 determines the distinct sensitivity of mTRPA1 and hTRPA1 to menthol

To systematically identify the molecular basis underlying the distinct sensitivity of mTRPA1 and hTRPA1 to menthol, we con- structed chimeras between the two channels. We first swapped three regions between mTRPA1 and hTRPA1: the cytoplasmic N terminus, the region spanning all transmembrane domains (TM1-TM6), and the cytoplasmic $\mathrm{C}$ terminus. The generated chimeras were expressed in $\mathrm{CHO}$ cells and assayed by calcium imaging. We found that swapping either the $\mathrm{N}$ terminus or the $\mathrm{C}$ terminus between mTRPA1 and hTRPA1 did not affect the menthol sensitivity of the host channel (data not shown). In contrast, hTRPA1-mTM1-6, a chimera constructed by introducing the transmembrane region from mTRPA1 into hTRPA1 (Fig. 3A), essentially recapitulated the bimodal sensitivity of mTRPA1 to menthol in $\mathrm{Ca}^{2+}$ imaging studies (Fig. $3 B, C$ ), whereas the reverse chimera mTRPA1-hTM1-6 behaved like hTRPA1 (Fig. $3 D, E)$. Specifically, as observed for wild-type mTRPA1, hTRPA1-mTM1-6 was not activated by $250 \mu \mathrm{M}$ menthol, displayed the off-response characteristic of mTRPA1 (Fig. $3 B$ ), and revealed no $\mathrm{MO}$ response in the presence of $250 \mu \mathrm{M}$ menthol (Fig. $3 C$ ). However, mTRPA1-hTM1-6 was effectively activated (Fig. $3 D$ ), but not inhibited by menthol (Fig. $3 E$ ). These data demonstrate that the molecular determinant for differential menthol modulation resides within the region of TRPA1 between the start of TM1 and the end of TM6.

To further narrow down the region/amino acids responsible for the differences observed between mTRPA1 and hTRPA1, we constructed the chimera hTRPA1-mTM1-4 by introducing the region including $\mathrm{mTRPA} 1 \mathrm{TM} 1$ through TM4 into hTRPA1 and the chimera hTRPA1-mTM5-6 by introducing the region including mTRPA1 TM5 through TM6 into hTRPA1 (Fig. 3A). Swapping TM1-4 between mouse and human TRPA1 was ineffective at switching menthol response (Fig. $3 B, C$ ), indicating that this region does not contribute to the differential phenotype. However there was a functional switch when TM5-6 was swapped. hTRPA1-mTM5-6 behaved like wild-type mTRPA1 in response to menthol (Fig. $3 B, C$ ). In the reverse swap, the menthol response of the chimera mTRPA1-hTM5-6 behaved as hTRPA1 (Fig. 3D,E). In FLIPR, mTRPA1-hTM5-6 was activated by menthol with an $\mathrm{EC}_{50}$ of $43.9 \pm 6.4 \mu \mathrm{M}$ and it was not significantly inhibited by menthol (Fig. 3F,G). However, hTRPA1-mTM5-6 showed a bimodal bell-shaped response to menthol activation and was completely inhibited by menthol with an $\mathrm{IC}_{50}$ of $71.0 \pm 16.5 \mu \mathrm{M}$ (Fig. $3 F, G$ ). These data demonstrate that the distinct menthol sensitivity of mTRPA1 and hTRPA1 is determined by the region including TM5 through TM6.

Sequence alignment of the region TM5 through TM6 revealed 17 nonidentical amino acids between mTRPA1 and hTRPA1 (Fig. 4A). To identify residues accounting for the different responses of mTRPA1 and hTRPA1 to menthol, we made miniature chimeras and point mutations that switched specific amino acids between mTRPA1 and hTRPA1. Characterizing these mu- 
A hTRPA1 $250 \mu \mathrm{M}$ Menthol
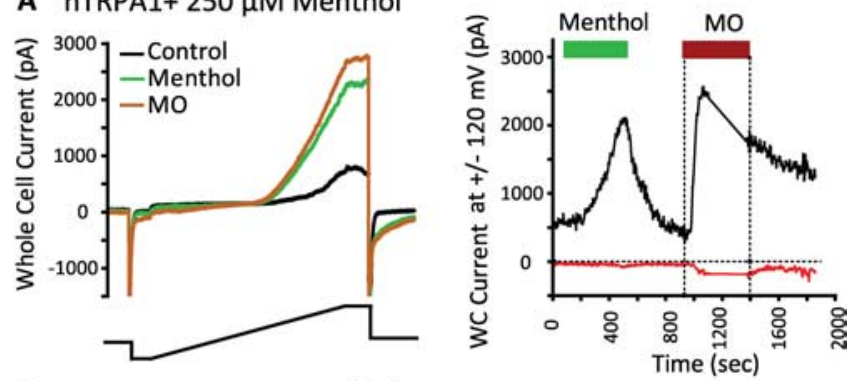

B mTRPA1 $+50 \mu \mathrm{M}$ Menthol
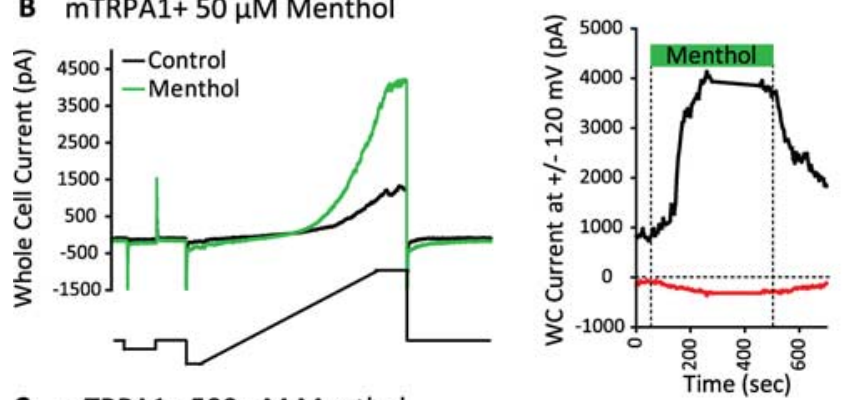

C mTRPA1 $+500 \mu \mathrm{M}$ Menthol
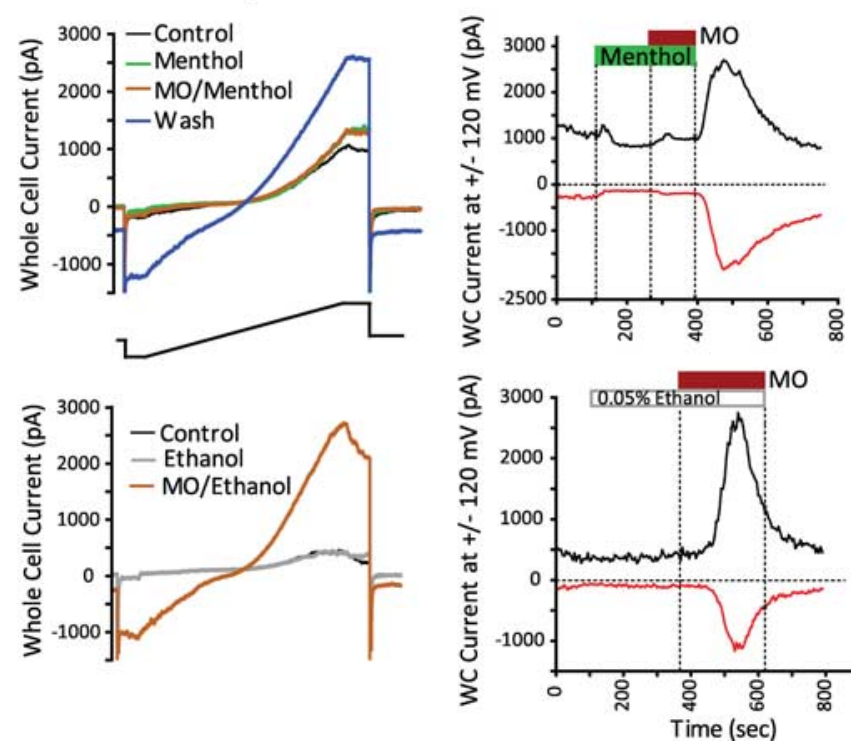

Figure 2. Menthol modulation of TRPA1 is species- and concentration-dependent as assayed by electrophysiology. $\boldsymbol{A}$, Menthol activates hTRPA1. Left, Both menthol ( $250 \mu \mathrm{M})$ and M0 $(100 \mu \mathrm{M})$ similarly increase whole-cell currents in HEK293T cells transiently transfected with hTRPA1. Representative current traces are shown before (black), at the peak of the menthol response (green), and at the peak of the M0 response (brown) after reversal of the menthol effect. Cells were challenged every $5 \mathrm{~s}$ with voltage ramps between -120 and $+120 \mathrm{mV}$ ( 2.8 $\mathrm{mV} / \mathrm{ms}$ ) from a holding potential of $-50 \mathrm{mV}$ (protocol shown below). The currents at -120 (red) and +120 (black) $\mathrm{mV}$ were measured and plotted over the entire time course of the experiment (right). Menthol and $\mathrm{MO}$ application is indicated by the green and brown bars, respectively. Vertical dashed lines indicate the times that either the indicated compound arrived at the bath or washout was initiated. Note that during the gap between 1100 and $1370 \mathrm{~s}$, the cell was challenged with voltage step protocols (extracellular saline, $2 \mathrm{~mm} \mathrm{Ca}^{2+} \mathrm{ES}$; pipette saline, $0 \mathrm{~mm} \mathrm{Ca}^{2+} \mathrm{IS}$ ). Responses of cells tested in $0 \mathrm{~mm} \mathrm{Ca}^{2+} \mathrm{ES}$ were similar to those obtained in $2 \mathrm{~mm} \mathrm{Ca}{ }^{2+} \mathrm{ES}$ and combined. $\boldsymbol{B}$, Low concentrations of menthol activate mTRPA1. Left, Menthol $(50 \mu \mathrm{m})$ increases whole-cell currents in HEK293T cells transiently transfected with mTRPA1. Representative current traces are shown before (black) and at the peak of the menthol response (green). Right, Exposure to menthol is indicated by the green bar. Note that during the gap between 220 and $380 \mathrm{~s}$, the cell was challenged with voltage step protocols (extracellular saline, $0 \mathrm{~mm} \mathrm{Ca}{ }^{2+} \mathrm{ES}$; pipette saline, $\left.0 \mathrm{~mm} \mathrm{Ca}^{2+} I S\right)$. C, High concentrations of menthol block subsequent responses to M0 $(100 \mu \mathrm{M})$ applied in the presence of menthol $(500 \mu \mathrm{M})$. Top left, Representative current traces are shown before (black), at the peak of the menthol response (green), at the peak of the $\mathrm{MO} /$ menthol response (brown), and at the peak of the washout response (blue). Top right, The whole-cell currents at -120 (red) and +120 (black) $\mathrm{mV}$ were tants by both calcium imaging and FLIPR revealed that the amino acid in TM5 predicted to be approximately one-third of the way along the $\alpha$ helix (G878 in mTRPA1 and V875 in hTRPA1) was a major contributor to the species-specific gating by menthol: hTRPA1-V875G displayed menthol sensitivity similar to that of mTRPA1, was activated by menthol in a bimodal manner, and was inhibited by menthol with an $\mathrm{IC}_{50}$ of $118.7 \pm 16.5 \mu \mathrm{M}$ (Fig. $4 B, C, F, G)$, although this $\mathrm{IC}_{50}$ value is right shifted by twofold to threefold compared with wild-type mTRPA1. These data demonstrate that V875 in TM5 of hTRPA1 (and the corresponding amino acid G878 in mTRPA1) is a major determining factor of sensitivity to menthol.

Three other mutants hTRPA1-L867F (TM5), hTRPA1L894F/P897A/S900T/I905L (pore helix), and hTRPA1-V942I/ S943A/I946M (TM6) showed subtle changes in response to menthol compared with hTRPA1 (supplemental Fig. 3, available at www.jneurosci.org as supplemental material). We next substituted all nine amino acids that contributed to a switch phenotype (Fig. $4 A$, residues shown in red) (hTRPA1-FGFATLIAM) and found that this channel recapitulated mTRPAl fully: hTRPA1FGFATLIAM was completely inhibited by menthol with an $\mathrm{IC}_{50}$ of $36.6 \pm 9.0 \mu \mathrm{M}$, similar to mTRPA1 (Fig. $4 E, G$ ), and its activation by menthol became bimodal (Fig. $4 D, F$ ). Together, these data demonstrate that nine residues from TM5-TM6 of hTRPA1 when replaced by the equivalent MTRPA1 amino acids completely switch the menthol-sensitivity to a mouse TRPA1-like phenotype.

To test whether the identified residues play a more general role in determining species-dependent modulation of TRPA1 by other chemicals, we examined the response of the mutant hTRPA1-FGFATLIAM to AMG5445, a compound reported to partially activate mTRPA 1 and inhibit hTRPA 1 (the reverse observed for menthol sensitivity) (Klionsky et al., 2007). Consistent with this previous report, AMG5445 activated mTRPA1 and its maximum efficacy was $\sim 50 \%$ of that of $100 \mu \mathrm{M} \mathrm{MO}$ (Fig. $4 H$ ), and completely inhibited hTRPAl (Fig. 4I). Importantly, the modulation by AMG5445 was switched in hTRPA1FGFATLIAM compared with wild-type hTRPA1 and behaved like wild-type mTRPA1 (Fig. $4 \mathrm{H}, \mathrm{I}$ ). These data demonstrate that residues at these positions govern the species-specific responses of TRPA1 not only to menthol but also to other compounds that reveal species specific effects as well.

\section{The TM5 domain determines menthol sensitivity of TRPA1}

Studying the chimeras of hTRPAl and mTRPAl led us to the identification of key residues accounting for the agonistic vs antagonistic effects of menthol. However, because both human and mouse TRPA1 respond to menthol, they might also share domains that are involved in menthol sensitivity. For example, it is possible that both human and mouse TRPA1 have a common menthol-binding site, which might translate into ion channel activation or inhibition based on the residues identified in the pore domain. Toward this end, we studied menthol sensitivity of nonmammalian TRPA1 orthologs, including dTRPA1. dTRPA1 has been shown to function differently from mammalian TRPA1

$\leftarrow$

measured and plotted over the entire time course of the experiment. The durations of exposure to menthol and M0 are indicated by the green and brown bars, respectively. Bottom left, Ethanol (0.05\%) had no effect on whole-cell conductance (gray trace) and $100 \mu \mathrm{m}$ M0 elicited a large response (brown trace). Bottom right, The currents at $\pm 120 \mathrm{mV}$ are shown over the course of the experiment (extracellular saline, $2 \mathrm{~mm} \mathrm{Ca}^{2+} E S$; pipette saline, $0 \mathrm{~mm} \mathrm{Ca}^{2+} I S$ ). 
A

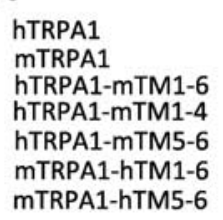

B
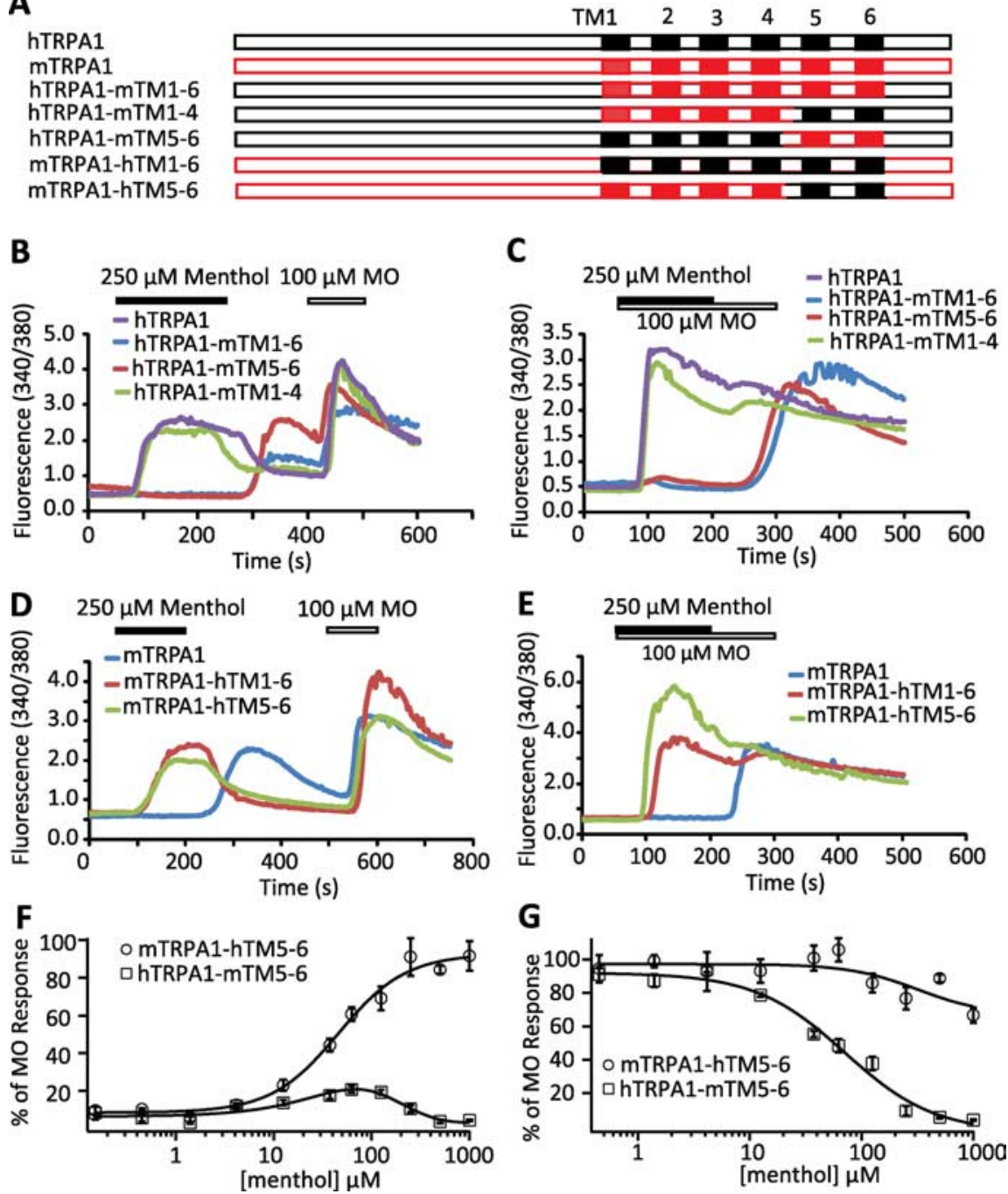

G

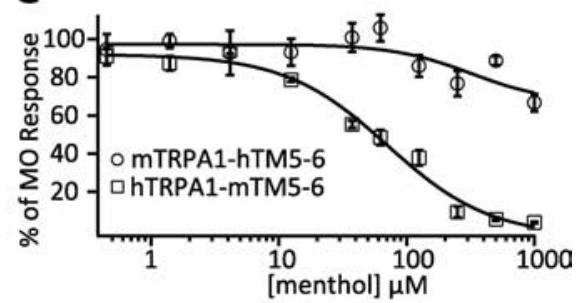

Figure 3. Determinants of the distinct responses of hTRPA1 and mTRPA1 to menthol reside in the region from TM5 to TM6. $\boldsymbol{A}_{\boldsymbol{t}}$ Schematic representations of $m$ TRPA1-hTRPA1 chimeras. The amino acid compositions of the indicated chimeras are described in the supplemental Table 1 (available at www.jneurosci.org as supplemental material). $\boldsymbol{B}-\boldsymbol{E}$, The responsiveness of hTRPA1 (purple traces), hTRPA1-mTM1- 6 (blue traces), hTRPA1-mTM5- 6 (red traces), and hTRPA1-mTM1- 4 (green traces) to menthol (activation) and menthol-dependent inhibition of $M 0$ activation (inhibition) is shown in $\boldsymbol{B}$ and $\boldsymbol{C}$, respectively, whereas the responsiveness of mTRPA1 (blue traces), mTRPA1-hTM1- 6 (red traces), and mTRPA1-hTM56 (green traces) to menthol activation and inhibition is displayed in $\boldsymbol{D}$ and $\boldsymbol{E}$, respectively. $\boldsymbol{F}$, Concentration-responsive relationships of menthol activation of hTRPA1mTM5- 6 (open squares) or mTRPA1-hTM5- 6 (open circles). $G$, Concentration- dependence curves of menthol inhibition of 100 $\mu \mathrm{M}$ M0-evoked hTRPA1-mTM5- 6 (open squares) or mTRPA1-hTM5- 6 (open circles) activity. Note that hTRPA1-mTM5-6 recapitulates the sensitivity of mTRPA1 to menthol, whereas mTRPA1-hTM5- 6 behaves like hTRPA1.

in that it is not activated by reactive compounds such as $\mathrm{MO}$, and it is activated by warm instead of cold temperatures (Viswanath et al., 2003).

We tested whether dTRPA1 heterologously expressed in HEK293T cells was functional using the whole-cell configuration of the patch-clamp technique. Raising the bath temperature from near $20^{\circ} \mathrm{C}$ to $33^{\circ} \mathrm{C}$ evoked outwardly rectifying currents (Fig. $5 \mathrm{~A}$ ) that reversed during cooling (Fig. 5B). In contrast, $250-500 \mu \mathrm{M}$ menthol was ineffective: the change in evoked current at +120 $\mathrm{mV}$ was not significant over $3 \mathrm{~min}$ of recording, similar to cells transfected with vector alone (Fig. $5 C$, leftmost bars). Similar results were obtained with as low as $2.5 \mu \mathrm{M}$ menthol throughout the voltage range studied (within the range of $25-100 \mu \mathrm{M}$ menthol: $-0.53 \pm 0.3 \mathrm{pA} / \mathrm{pF}$ at $+120 \mathrm{mV} ; n=7$ ). Furthermore, the presence of $500 \mu \mathrm{M}$ menthol during the warming protocol did not significantly depress the warminduced currents compared with those in the presence of vehicle alone $(0.05 \%$ ethanol) (Fig. 5C, dTRPA1/Menthol vs dTRPA1/Ethanol). These data indicate that whereas heating is able to activate dTRPA1, menthol elicits no detectable increase in conductance and dTRPA1mediated warm-induced currents are not significantly suppressed or enhanced by menthol.

We reasoned that chimeras of mammalian and Drosophila TRPA1 channels might allow us to identify the conserved molecular determinants of menthol sensitivity of mTRPA1 and hTRPA1 that are not present in dTRPAl. The chimera dTRPA1-mN, in which the mTRPA1cytoplasmic $\mathrm{N}$-terminal 720 amino acids of MTRPAl is exchanged for the $\mathrm{N}$ terminus of dTRPA1 (supplemental Table 1, supplemental Fig. 1, available at www. jneurosci.org as supplemental material), responded to $\mathrm{MO}$ with an $\mathrm{EC}_{50}$ of $103.2 \pm$ $26.5 \mu \mathrm{M}$ and an efficacy comparable with mTRPA1 (supplemental Table 2, available at www.jneurosci.org as supplemental material). In contrast, menthol was ineffective at this chimera (supplemental Fig. 5, available at www.jneurosci.org as supplemental material), suggesting that the molecular determinant of menthol resides in the TM-spanning or $\mathrm{C}$ terminus domains of mTRPA1. Strikingly, the chimera mTRPA1-dTM5, which contains the TM5 domain from dTRPA1 in mTRPA1 background, completely lost menthol sensitivity while retaining normal MO responsiveness. In calcium imaging, $250 \mu \mathrm{M}$ menthol neither activated mTRPA1-dTM5 nor produced an off response during its washout (Fig. $5 D$, blue trace). Furthermore, the inhibitory effect of high concentrations of menthol on MO responsiveness was abolished (Fig. $5 E$, blue trace). These results were corroborated in FLIPR experiments (Fig. $5 F, G$ ). Consistent with the profound effect of dTM 5 on mTRPA 1 menthol sensitivity, insertion of dTM5 into hTRPA1 abolished menthol dependent activation (Fig. 5D-G). The insertion of dTM5 into hTRPA1 did not alter the $\mathrm{MO} \mathrm{EC}_{50}$ (supplemental Table 2, available at www.jneurosci.org as supplemental material), although it appears to enhance the decay of $\mathrm{MO}$-evoked $\mathrm{Ca}^{2+}$ responses (Fig. $5 D, E$ ).

We further characterized the two dTM5-containing chimeras using electrophysiological methods. mTRPA1-dTM5 revealed qualitatively similar voltage dependence of activation to that reported previously for mTRPA1 (supplemental Fig. 4A, B, available at www.jneurosci.org as supplemental material) and the current elicited by a step to $+180 \mathrm{mV}$ could be used to normalize compound sensitivity. Swapping in dTM5 completely abolished the activation of mTRPA1 by $50 \mu \mathrm{M}$ menthol (Fig. $5 \mathrm{H}$ ). Furthermore, the inhibition of mTRPA1 at high concentrations of men- 
thol (Fig. 5I, leftmost bars) was abolished in the mTRPA1-dTM5 chimera (Fig. 5I, rightmost bars; supplemental Fig. 4D,E, available at www.jneurosci.org as supplemental material); $\mathrm{MO}$ responses were similar to those treated with vehicle alone (Fig. 5I, rightmost bars; supplemental Fig. $4 F$, available at www.jneurosci.org as supplemental material).

Consistent with a critical role for TM5 in menthol responsiveness, the activation of human TRPA 1 by $250 \mu \mathrm{M}$ menthol was completely abolished in the hTRPA1dTM5 chimera (Fig. 5J). It should be noted that the current amplitudes of hTRPA1-dTM5 in response to either a voltage step to $+180 \mathrm{mV}$ (supplemental Fig. $4 K$ ) or $100 \mu \mathrm{M} \mathrm{MO} \mathrm{(supplemental} \mathrm{Fig.}$ $4 L$, available at www.jneurosci.org as supplemental material) were significantly lower than those of hTRPA1 and may be caused by expression levels, and/or faster desensitization/inactivation kinetics of this chimera (supplemental Fig. 4G-J, available at www.jneurosci.org as supplemental material). Collectively, these data demonstrate that the TM5 domain is required for menthol sensitivity of mammalian TRPA1.

None of the other mouse and fly TRPA1 chimeras responded to any stimulus, suggesting that these channels are either not properly expressed, or structurally compromised (supplemental Fig. 1, available at www.jneurosci.org as supplemental material). We further attempted to introduce mTM56, hTM56, mTM5, and hTM5 to the chimera dTRPA1-mN (supplemental Fig. 1, available at www. jneurosci.org as supplemental material). However, none of these chimeras responded to MO or menthol.

\section{Specific residues in TM5 are required for menthol sensitivity of TRPA1}

The amino acid sequence of dTM5 is poorly conserved with that of mTM5 or hTM5, particularly in the predicted cytoplasmic side of TM5 (Fig. $6 \mathrm{~A}$ ). We made a chimera by introducing the divergent part of dTM5 to hTRPA1 (hTRPA1-dTM5a). Calcium imaging results demonstrate that hTRPA1-dTM5a revealed no menthol activation (Fig. 6B, C,). Within this stretch of 16 aa, 7 of them are conserved between mTRPA1 and hTRPA1, but divergent from dTRPA1 (Fig. 6A, residues highlighted in yellow). We made four mutants to substitute these seven amino acids in mTRPA1. These mutants included mTRPA1-K871Q, mTRPA1-S876V/T877L, mTRPA1-I881S/F882I, and mTRPA1-L884I/L885I. Calcium imaging revealed that mTRPA1-K871Q and mTRPA1-I881S/ F882I did not respond to MO (potentially nonfunctional), whereas mTRPA1-L884I/L885I responded to both MO and menthol (data not shown). Strikingly, mTRPA1-S876V/T877L com-
TM5 Pore Helix Selectivity Filter TM6

MTRPA1 869 IFKTLLRSTGVIFLLLAFGLSFYVLLNFDAFSTPLLSLIQTFSMMLGINYRDAFLEPLFRNELAYPVLTFGLIAFTMF 950 hTRPA1 866 ILKTLLRSTVVFIFLLLAFGLSFYILLNLQDPFSSPLLSIIQTFSMMLGDINYRESFLEPYLRNELAHPVLSFAQLVSFTIF 947
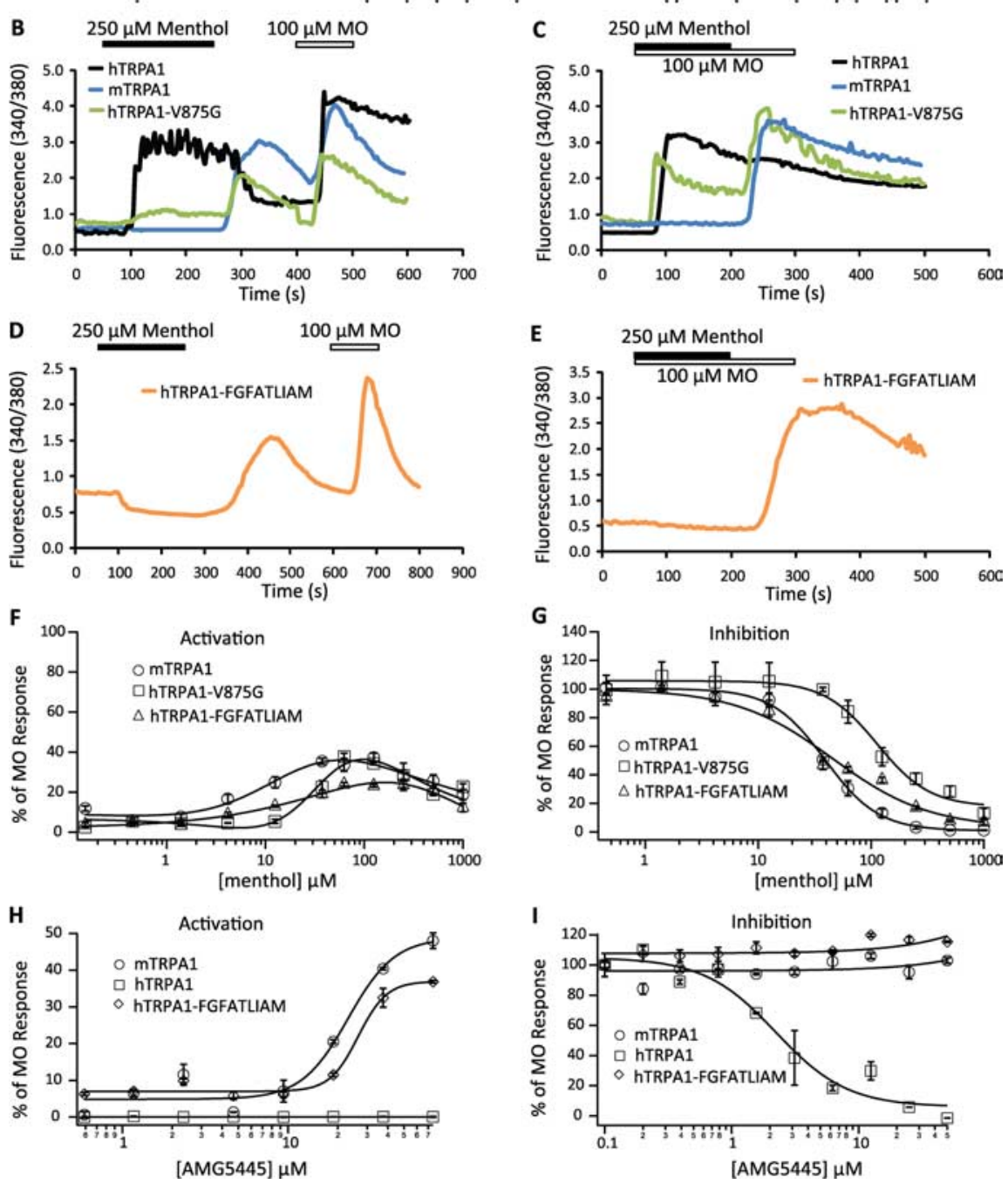

Figure 4. Residues within TM5-TM6 govern species-specific response of TRPA1 to menthol and AMG5445. $\boldsymbol{A}$, Sequence alignment of the region from TM5 to TM6 reveals a total of 17 nonidentical amino acids (red and blue) in mTRPA1 and hTRPA1. Residues shown in red are responsible for the distinct responses of mTRPA1 and hTRPA1 to menthol and AMG5445; those in blue did not alter the host channel response to menthol. The predicted positions of TM5, pore helix, selectivity filter, and TM6 (the rest of TM6 is identical between mTRPA1 and hTRPA1 and, thus, is not shown) are also depicted. $\boldsymbol{B}, \boldsymbol{C}$, Calcium imaging experiments pronounced off response, green trace) $(\boldsymbol{B})$ and inhibition ( $\boldsymbol{C}$. D, E, hTRPA1-FGFATLIAM recapitulates the sensitivity of mTRPA1 to menthol activation $(\boldsymbol{D})$ and inhibition $(\boldsymbol{E})$. $\boldsymbol{F}, \mathbf{G}$, Concentration- effect relationships of mTRPA1 (open circles), hTRPA1-V875G (open squares), and hTRPA1-FGFATLIAM (open triangles) to menthol activation and inhibition. $\boldsymbol{H}$, Concentration-response relationships of mTRPA1 (open circles), hTRPA1 (open squares), and hTRPA1-FGFATLIAM (open diamonds) to AMG5445. Note that AMG5445 acts as a partial agonist at MTRPA1 and hTRPA1-FGFATLIAM and has no agonist activity at hTRPA1. I, Concentrationresponse relationships of AMG5445 inhibition of $30 \mu \mathrm{M} M 0$-induced responses mediated by mTRPA1 (open circles), hTRPA1 (open squares), and hTRPA1-FGFATLIAM (open diamonds) activity. Note that AMG5445 is a full antagonist at hTRPA1.

pletely lost its menthol sensitivity while retaining a relatively normal MO response (supplemental Table 2, available at www. jneurosci.org as supplemental material) as assayed by both calcium imaging and FLIPR (Fig. 6B-E). We also made the corresponding mutant in hTRPA1 (hTRPA1-S873V/T874L, in which the corresponding Ser-873 and Thr-874 of hTRPA1 were mutated to Val and Leu of Drosophila). Whereas the MO induced responses of hTRPA1-S873V/T874L were normal (supplemental Table 2, available at www.jneurosci.org as supplemental material), the menthol sensitivity was severely reduced compared with hTRPA1 (Fig. 6B-E, Table 1). 

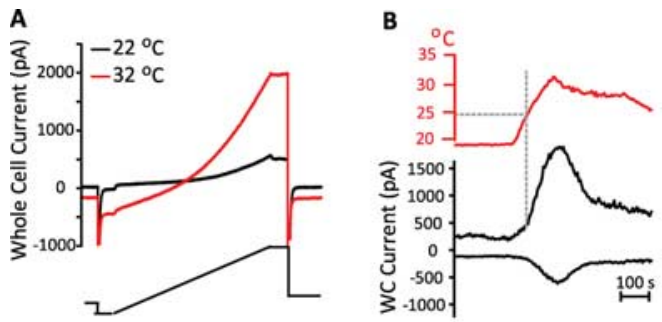

\section{C}
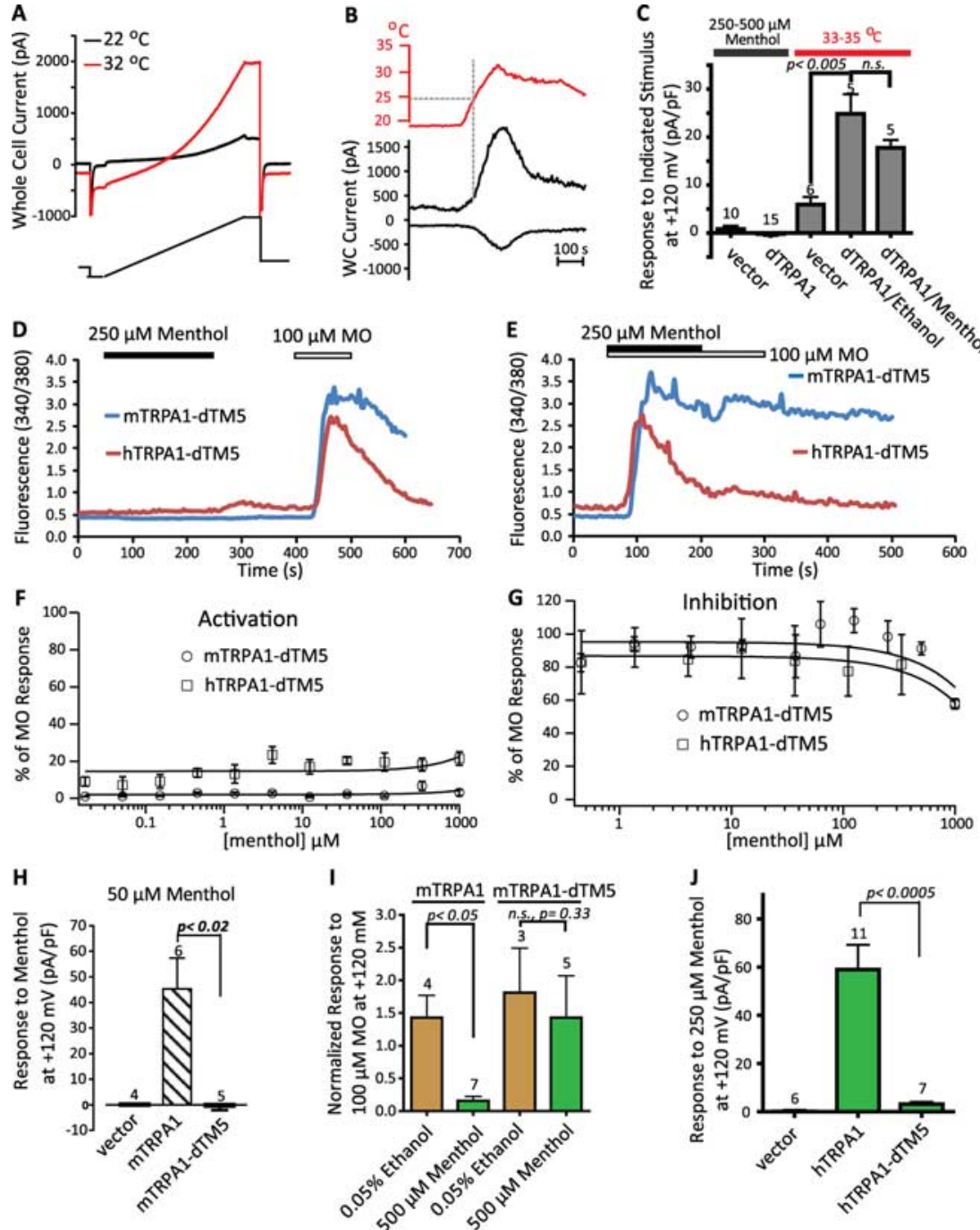

Figure 5. Warm-activated Drosophila TRPA1 is insensitive to (-)-menthol, and replacement of mammalian TM5 with dTM5 abolishes modulation of mammalian channels by menthol. $\boldsymbol{A}$, A dTRPA1-transfected cell was challenged with a voltage ramp (from -120 to $+120 \mathrm{mV}$ ) at $22^{\circ} \mathrm{C}$ (black trace) and $32^{\circ} \mathrm{C}$ (red trace). The warming-induced current was -35.5 and $+85 \mathrm{pA} / \mathrm{pF}$ at -120 and $+120 \mathrm{mV}$, respectively. This cell revealed no activation by menthol (at either 25 or $250 \mu \mathrm{m} ; 0 \mathrm{~mm} \mathrm{Ca}^{2+} \mathrm{ES} / 0 \mathrm{~mm} \mathrm{Ca}{ }^{2+} \mathrm{IS}$ ). $\boldsymbol{B}$, Time course of currents measured at $-120 \mathrm{mV}$ and $+120 \mathrm{mV}$ before and during warming and recovery from warming for the cell shown in $A$. Heating-induced currents often revealed apparent desensitization. WC, Whole cell. C, dTRPA1 is neither activated by $250-500 \mu \mathrm{m}$ nor inhibited by $500 \mu \mathrm{m}$ menthol. The two leftmost bars indicate the change in whole-cell current density at $+120 \mathrm{mV}$ during exposure to $250-500 \mu \mathrm{m}$ menthol at $\sim 20-24^{\circ} \mathrm{C}$ for the number of vector or dTRPA1-transfected cells shown above each bar. Similar results were obtained for cells recorded with or without external calcium, or in $0 \mathrm{~mm} \mathrm{Ca}{ }^{2+}$ - or $100 \mathrm{~nm} \mathrm{Ca}^{2+}$-containing intracellular saline, and were combined. Cells were exposed to menthol for at least 3 min, which was at least three times the latency of menthol to activate $h$ TRPA1 under similar conditions. The set of gray bars are the means $\pm S E M$ for the increases in current density at $+120 \mathrm{mV}$ during heating to $33-35^{\circ} \mathrm{C}$ from $20-22^{\circ} \mathrm{C}$. Similar results were obtained when currents were measured at $-120 \mathrm{mV}$ (data not shown). Whereas control HEK293T cells ("vector") revealed a heating-induced increase in conductance, the response of dTRPA1-transfected cells is significantly larger. The threshold for activation by warming was near $27^{\circ} \mathrm{C}$, as described previously for dTRPA1 expressed in oocytes (Viswanath et al., 2003). To determine whether menthol could inhibit dTRPA1-mediated warm responses, the heat stimulus was applied in the presence of either $500 \mu \mathrm{m}$ menthol or $0.05 \%$ ethanol (dTRPA1/Ethanol vs dTRPA1/Menthol) in the presence of external calcium. Although a slight suppression was observed in the presence of menthol, the effect was not significant. Only dTRPA1 cells that revealed heatresponses at least2SDs larger than the vector control heat response were included in this analysis. YFP-positive cells were tested up to $5 \mathrm{~d}$ after transfection with vector alone or dTRPA1. D, E, Menthol ( $250 \mu \mathrm{M}$ ) neither activated (D) nor inhibited (E) both mTRPA1-dTM5 (blue trace) and hTRPA1-dTM5 (red trace) as assayed by ratiometric calcium imaging. $\boldsymbol{F}, \boldsymbol{G}$, Concentration-dependence curves of menthol activation $(\boldsymbol{F})$ and inhibition $(\boldsymbol{G})$ of mTRPA1-dTM5 (open circles) and hTRPA1-dTM5 (open squares). $\boldsymbol{H}$, In whole-cell voltage clamp, mTRPA1-dTM5 was not activated during 3 min exposure to $50 \mu \mathrm{mat} \sim 25^{\circ} \mathrm{C}$ and was comparable to vector-transfected cells. The response of wild-type mTRPA1 to $50 \mu \mathrm{m}$ menthol is significantly greater. I, mTRPA1-dTM5 is not inhibited by high concentrations of menthol. The peak mTRPA1-dTM5 whole-cell current response to $100 \mu \mathrm{mM} 0$ was not significantly affected by the presence of menthol (500 $\mu \mathrm{m}$; right set of bars, green) compared with ethanol $(0.05 \%$; brown). Data from wild type (left bars) are included for comparison. The analysis was as described in Figure 2 . Examples of $\mathrm{MO}$-induced responses in the presence of $\mathrm{MO}$ or vehicle are presented in supplemental Figure $4 B$ (available at www.jneurosci.org as supplemental material). Conclusions were identical when data were expressed as M0 response normalized to cell size.J, hTRPA1-dTM 5 is not activated by menthol. There was no significant effect of $250 \mu \mathrm{m}(-)$-menthol on cells expressing hTRPA1-dTM5 compared to wild type. Values were not significantly different from vector-transfected cells $(p>0.1)$.
Sequence alignment of TRPA1 from different species revealed that TRPA1 from Anopheles gambiae (mosquitoTRPA1) and Takifugu (fugu-TRPA1), like dTRPA1, do not have Ser and Thr at these two positions (Fig. $6 F$ ). In line with the importance of Ser and Thr at these positions in determining menthol sensitivity, we found that mosquito-TRPA1 and fuguTRPA1 were not sensitive to menthol (supplemental Fig. 5, available at www. jneurosci.org as supplemental material), but were activated by $\mathrm{MO}$, albeit with lower affinity than mouse or human TRPA1 (supplemental Table 2, available at www.jneurosci.org as supplemental material). Thus, Ser and Thr at these two positions critically determine the menthol sensitivity of TRPA1.

The molecular determinants of menthol determines the sensitivity of TRPA1 to other TRPA1 modulators

To test whether the residues critical for menthol sensitivity may serve as more general determinants of TRPA1 modulation, we examined the responses of wild-type or mutant hTRPA1 to a series of compounds known to modulate TRPA1 (Table 1). Thymol, a natural compound found in thyme, is structurally related to menthol, and has essentially the same effect on mTRPA1 (Karashima et al., 2007) and hTRPA1 (Lee et al., 2008) as menthol. Not surprisingly, we found that replacing dTM5 into hTRPA1 nearly abolished the response to thymol, and hTRPA1-S873V/ T874L was poorly responsive (Table 1 ). FTS, a lipid compound, activated both hTRPA1 and hTRPA1-S873V/T874L, but failed to activate hTRPA1-dTM5, suggesting that the determinants of FTS sensitivity also reside in the TM5 domain but may differ from those of menthol. In contrast, there was no effect on activation of these mutants by trinitrophenol or MO (Table 1). AP18, AMG5445 and HC030031 have been reported to be hTRPA1 antagonists. Strikingly, we found that AP18 and AMG5445 did not inhibit the MO responses of hTRPA1-dTM5 and hTRPA1S873V/T874L. However, the responses of these mutants to ruthenium red, an external pore blocker, and HC030031 remained normal (Table 1).

We further tested AP18 on mTRPA1 and its mutants. AP18 inhibited $30 \mu \mathrm{M}$ MO-induced mTRPA1 activity (Fig. $7 A, B)$. The $\mathrm{IC}_{50}$ was $0.69 \pm 0.09 \mu \mathrm{M}$ as assayed by FLIPR. In contrast, the inhibitory effect of AP18 was totally abolished in the mutant mTRPA1-S876V/T877L (Fig. $7 A, B)$. It should be noted that AP18 is not 
a cysteine reactive compound as it did not form a glutathione adduct in vitro (supplemental Fig. 6, available at www.jneurosci. org as supplemental material).

Chen et al. (2008) proposed recently that electrophilic compounds like CMP1, a close analog of AMG5445, activate mTRPA1 and inhibit hTRPA1 through covalent modifications of cysteine residues in the $\mathrm{N}$ terminus of these channels (Chen et al., 2008). Interestingly, we found that the partial agonistic effect of AMG5445 was totally abolished in the mutant mTRPA1-S876V/T877L (Fig. 7C). Furthermore, the mutant hTRPA1-S873V/ T874L lost inhibition by AMG5445 (Fig. $7 D)$. These data demonstrate that the sensitivity of mTRPA1 and hTRPA1 to AMG5445 is also determined by Ser and Thr at these two positions.

\section{Is TM5 domain a binding site for} menthol and other chemicals?

To explore whether a binding pocket for menthol could be potentially formed by TM5, we modeled the pore module of mTRPA1 (including TM5, the linker between TM5 and TM6, and TM6) taking the structure of KcsA as a template. SMMLGD of mTRPA1 has the potential to form the selectivity filter of mTRPA1 based on sequence similarity to KcsA (TVGYGD) and the role of the aspartic acid residue (D918) in TRPA1 calcium selectivity (T. J. Jegla, unpublished data) (Fig. 8A). We chose KcsA over Kv1.2 as the template because the sequence around the predicted selectivity filter of mTRPA1 does not align with Kv1.2. The optimized mTRPA1 homology model was used for molecule docking calculations. These studies revealed a potential putative menthol binding pocket formed by TM5 (Fig. 8). Interestingly, the experimentally identified residues Thr-877 and Gly-878, which are critical for menthol sensitivity of MTRPA1, are predicted to be located within the pocket (Fig. $8 B, C$ ). According to this model, Gly-878 (which was identified as one of the major determinants of distinct mouse-human menthol response) makes extensive hydrophobic contacts with menthol, and Thr-877 (important for menthol sensitivity that is conserved between mouse and human) may form a hydrogen bond with the molecule. Furthermore, in line with the importance of Ser-876 and Thr-877 in determining mTRPA1 inhibition by AP18, AP18 is also able to dock into the menthol pocket and potentially forms a hydrogen bond with Thr-877 (Fig. $8 D$ ). These modeling results put forward the hypothesis that TM5 contributes directly to a binding pocket. However, the apparent consistencies between experimental and modeling results by no means constitute evidence for binding. Indeed, this model should be considered cautiously given the limited sequence homology between mTRPA1 and KcsA (Fig. 8A). not in other species.
TM5a

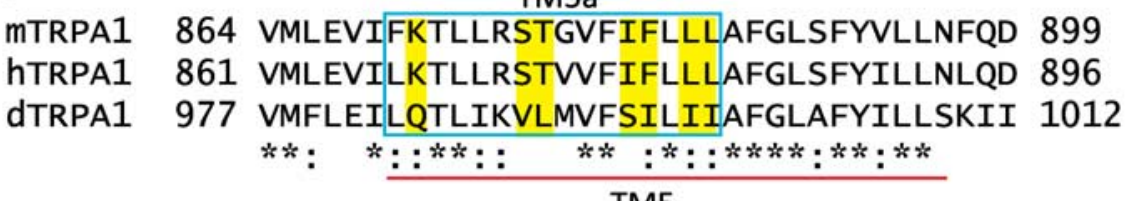

TM5
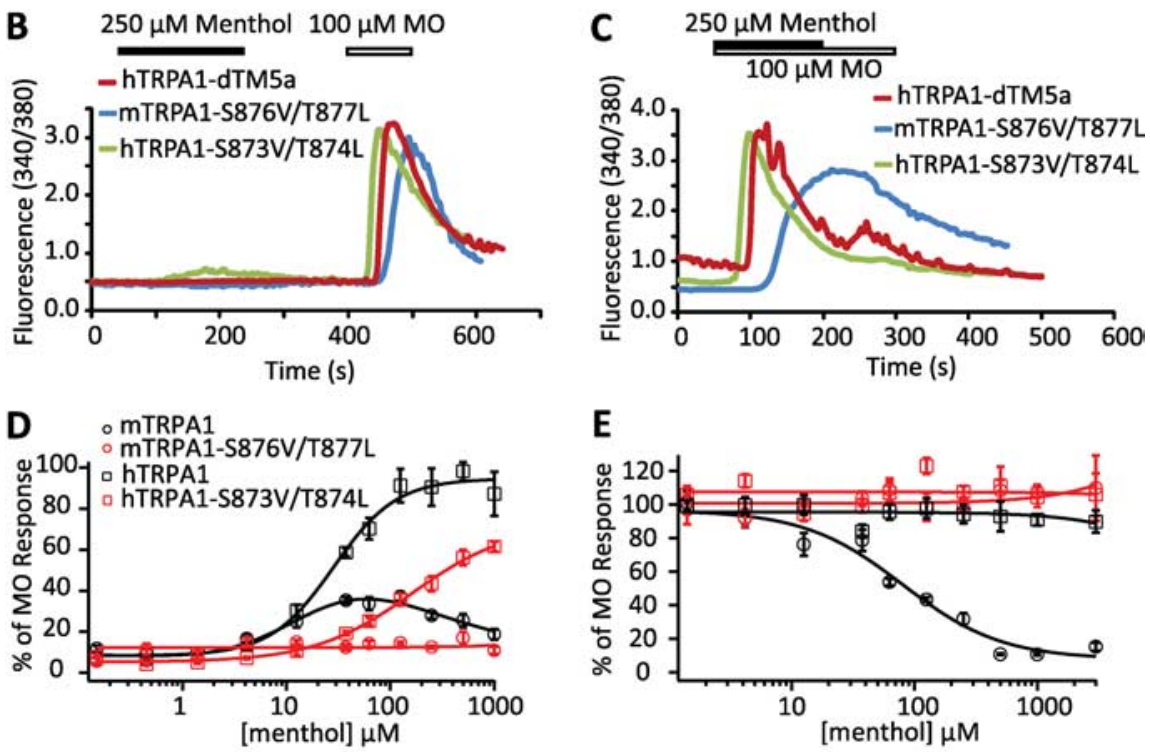

Figure 6. Specific residues in TM5 determine menthol sensitivity of TRPA1. $A$, Sequence alignment around the TM5 domain of mTRPA1, hTRPA1, and dTRPA1. The blue box (TM5a) indicates the region that was swapped from dTRPA1 into hTRPA1 (hTRPA1dTM5a). Residues highlighted by yellow within the TM5a region are identical between mTRPA1 and hTRPA1 and divergent from dTRPA1. $\boldsymbol{B}, \boldsymbol{C}$, The chimera hTRPA1-dTM5a ( $\boldsymbol{B}, \boldsymbol{C}$, red traces) was unresponsive to menthol. mTRPA1-S876V/T877L (blue traces) T874L by $250 \mu \mathrm{m}$ menthol was minimal (green trace, $\boldsymbol{B}) . \boldsymbol{D}, \boldsymbol{E}$, Concentration-dependence curves of menthol activation $(\boldsymbol{D})$ and (black open circles), mTRPA1-S876V/T877L (red open circles), hTRPA1 (black open squares), and fugu, fly, and mosquito reveals that the Ser and Thr residues critical for menthol sensitivity are conserved in mammalian TRPA1 but

\section{Discussion}

Menthol is best known to elicit a cooling sensation in humans (Eccles, 1994). In mice, TRPM8 has been demonstrated recently to underlie temperature sensation at cool temperatures (Bautista et al., 2007; Colburn et al., 2007; Dhaka et al., 2007). In addition to its cooling properties, menthol can produce a burning and painful sensation (Green, 1992). The molecular mechanism underlying this pronociceptive effect remains unknown. Here, we report that menthol is an efficacious agonist of human TRPA1, suggesting that TRPA1 could contribute to the menthol-induced burning sensation in humans. Furthermore, we found striking species-specific variation of menthol effects on TRPA1. In contrast to human TRPA1, TRPA1 orthologs from fly, mosquito, and fugu are insensitive to menthol, whereas murine TRPA1 reveals a bell-shaped concentration dependence as previously reported (Karashima et al., 2007). Although the biological significance of acquiring menthol sensitivity 

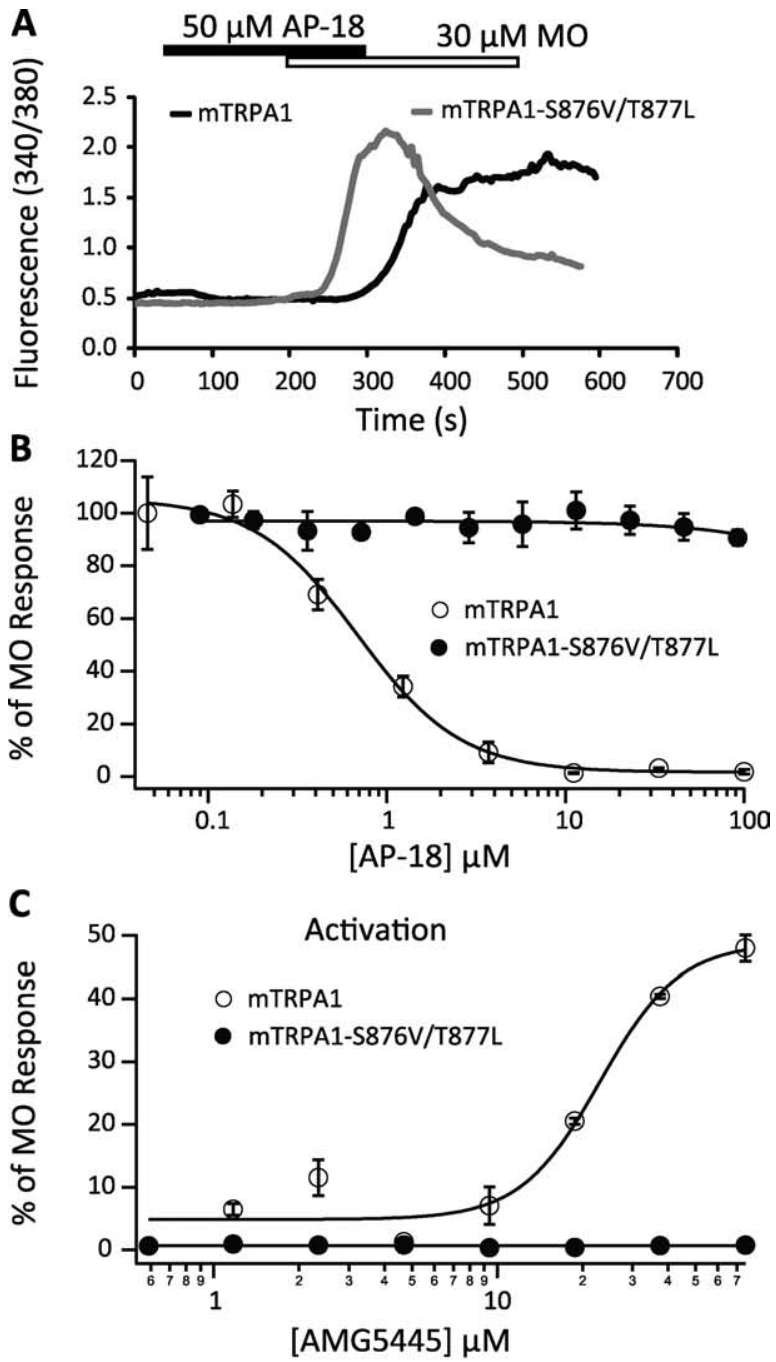

D

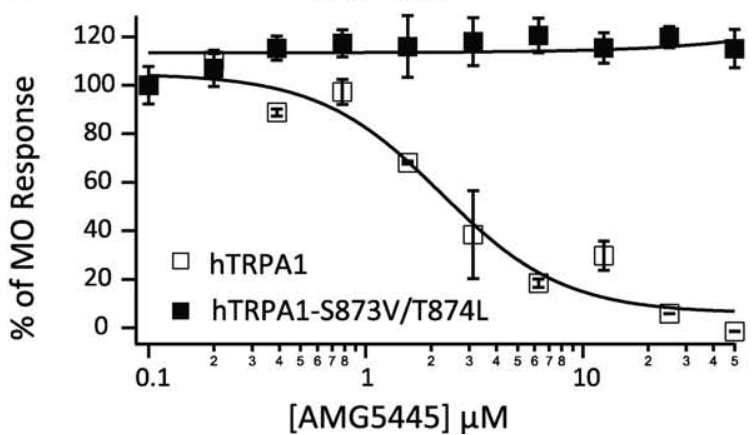

Figure 7. Residues critical for menthol sensitivity determine the sensitivity of TRPA1 to AP18 and AMG5445. A, AP18 $(50 \mu \mathrm{m})$ inhibited M0 $(30 \mu \mathrm{m})$-evoked response in cells transfected with mTRPA1 (black trace), but not in cells transfected with mTRPA1-S876V/T877L (gray trace). $\boldsymbol{B}$, Concentration-dependence curves of AP18 inhibition of mTRPA1 (open circles) or mTRPA1S876V/T877L (filled circles) activity evoked by $30 \mu \mathrm{M}$ MO. C, Concentration-response relationships of activation of mTRPA1 (open circles) and mTRPA1-S876V/T877L (filled circles) by AMG5445. D, Concentration- dependence curves of AMG5445 inhibition of $30 \mu \mathrm{m}$ M0-evoked $\mathrm{Ca}^{2+}$ responses in cells transiently transfected with either hTRPA1 (open squares) or hTRPA1S873V/T874L (filled squares).

(with different pharmacological profiles) in mammalian TRPA1 remains unclear, the unique menthol response profiles of various TRPA1 orthologs enabled a chimeric structure-function approach from which three major conclusions can be drawn. First, the TM5 domain critically determines the ability of TRPA1 to sense menthol. Second, switching nine pore domain residues in human TRPA1 to the mouse residues reverses how hTRPAl responds to menthol, turning menthol from a pure agonist to an apparent antagonist. Third, the identified structural domains required for menthol sensitivity also determine the sensitivity of mammalian TRPA1 to an array of TRPA1 modulators, including thymol, FTS, AP18, and AMG5445.

Initial studies identified menthol as an inhibitor of mTRPA1 when applied at high concentrations $(\geq 250 \mu \mathrm{M})$ (Macpherson et al., 2006). Subsequent studies further identified agonist activity of menthol at low concentrations (Karashima et al., 2007). The mechanisms underlying this bimodal effect of menthol on mTRPA1 have not been determined. Nilius and colleagues (Karashima et al., 2007) have proposed that menthol may have two separate binding sites on MTRPA1: one responsible for activation, the other for inhibition (Karashima et al., 2007). An alternative interpretation postulates a single binding site where block is observed as a consequence of activation. It is possible that menthol at high concentrations initially activates mTRPA1 but the channel rapidly switches into a nonconducting state (e.g., inactivation state), which has access to the open state after washout of menthol (off response). The response of mTRPA1 to menthol is reminiscent of voltage dependent activation of human ether-a-go-go-related gene (hERG) potassium channels: strong depolarizations cause hERG to transit through the open state to an inactivated state and, after repolarization, return to the open state before closing (Sanguinetti et al., 1995; Trudeau et al., 1995).

Regardless of the complexity of menthol effects on mTRPA1, it is striking that mTRPA1-dTM5 and mTRPA1-S876V/T877L are neither activated nor inhibited by menthol. How could specific residues in the TM5 domain determine the complicated menthol sensitivity of mouse TRPA1? One hypothesis is that the TM5 domain may constitute a binding site for menthol. Our modeling and docking studies reveal a putative menthol-binding site in TM5 regions. However, it is important to note that there is no direct evidence that menthol specifically binds to TRPA1. It is entirely possible that the effects of menthol are indirect, through modifications of the lipid bilayer, for example. At present, a cross-linkable menthol analog does not exist, and thus the sites of contact with the TRPA1 channel cannot be directly identified.

The existence of a ligand binding pocket in TM5 appears to be inconsistent with the general observation that most thermoTRPs have putative ligand binding sites within TM2 through TM4, particularly TM2 (Jordt and Julius, 2002; Vriens et al., 2004; Gavva et al., 2005; Voets et al., 2007). Interestingly, Bandell et al. (2006), using a high-throughput random mutagenesis screen, identified Tyr-745 in TM2 as a critical determinant of menthol sensitivity of mTRPM8. Given this precedence, it is possible that the region from TM2 to TM4 in TRPA1 may contain a common binding site for nonreactive TRPA1 modulators, whereas the TM5 domain is involved in translating initial binding to channel gating. To test whether a conserved role of tyrosine in determining menthol sensitivity exists in mTRPA1, we individually mutated the two tyrosine residues (Tyr788 and Tyr-875) located in the TM2 and TM3 of mTRPA1 to alanine. We found that these mutations do not affect mTRPA1 menthol sensitivity (data not shown). We also made a series of mTRPA1dTRPA1 chimeras. Unfortunately, most of these resulted in nonfunctional channels, and specific involvement of TM2-TM4 in menthol sensitivity could not be addressed. However, we are able to conclude that the TM1-TM4 region is not involved in the distinct responses of mTRPA1 and hTRPA1 to menthol. Regardless of its 
direct involvement in menthol binding or in gating, the TM5 domain critically determines the ability of TRPA1 to sense menthol.

How could TM5 mechanistically determine the menthol sensitivity of TRPA1? TRPA1 is predicted to have a similar structure as voltage-gated $\mathrm{K}^{+}$channels in which TM5 forms the outer helix of the pore and the TM4-TM5 linker helix is critical for electromechanical coupling by opening or closing the gate located in TM6 (Long et al., 2005). In both mammalian TRPA1 channels, Ser and Thr residues located in the predicted inner side of TM5 were identified as critical for the sensitivity of mouse (and to a large extent, human) TRPA1 to menthol. Perhaps important for sensitivity to menthol as well as the gating phenotype of methanol, the Ser and Thr residues are followed by a Gly residue (G878) in mTRPA1. Interestingly, all of these amino acids are known to break $\alpha$-helical symmetry (Levitt and Greer, 1977; Levitt, 1978). Given the properties of these residues, it is possible that TM5 may form a hinge at this location. In line with this idea, our molecular dynamic simulation of mTRPA1 reveals a kink in TM5 helix at these Ser and Thr residues in mammalian TM5. Thus, it is conceivable that either direct menthol interactions or conformational changes sensed by these residues after menthol binding to distant regions could be transmitted to TM6, leading to channel gating. The residues at these positions in TRPA1 from nonmammalian species are $\alpha$-helix favoring ( $\mathrm{Val}$ and Leu), which may preclude this proposed mechanism. Interestingly, Grimm et al. (2007) have found that a naturally occurring helixbreaking mutation in TM5 (Ala-419-Pro) of TRPML3 leads to constitutive channel activation. They further demonstrated that the inner one-third of TM5 from TRPML1, TRPML2, TRPV5, and TRPV6 is highly susceptible to proline-based kinks. Together with the present study, these data suggest that TM5 may play a critical role in TRP channel function.

Not only have we identified residues crucial for modulation of mammalian TRPA1 by menthol, we also identified nine residues from the pore module that specifically accounts for the speciesspecific responses of TRPA1 to menthol (activator vs inhibitor) (Fig. $8 \mathrm{~A}$, Gly878, amino acids highlighted in red). It is possible that Gly878 immediately after Ser-Thr in mouse TM5 makes the hinge too floppy to confer full agonism in mouse TRPA1 and/or it alters other properties of the pore region that then confers the blocking effect of menthol at high concentrations. In support of this hypothesis, hTRPA1-V875G revealed a menthol "inhibitory phenotype," albeit incomplete. Interestingly, Chen et al. (2008) found recently that CMP1, an electrophilic reactive compound, activates mTRPA1 but inhibits hTRPA1. They identified the same residues in the upper part of TM6 as determinants of species-specific gating. Based on their findings, they proposed that covalent modification of TRPA1 can lead to different functional consequences of the channel. However, it is not clear how CMP1 could inhibit MO-activated hTRPA1 if both CMP1 and MO act through covalent modifications of cysteine residues. Chen et al. (2008) argued that the covalent modification of cysteines in TRPA1 by MO is rapidly reversible. However, the half-life of isothiocyanate-cysteine adducts was reported to be in the order of approximately $1 \mathrm{~h}$ at physiological $\mathrm{pH}$ and temperature (Conaway et al., 2001). Furthermore, the successful labeling of MO alkyne adducts on TRPA1 by click chemistry also suggests that $\mathrm{MO}$ adducts remain rather stable because the click reaction itself takes $1 \mathrm{~h}$ to complete (Macpherson et al., 2007a). Given these concerns, we raise another possibility: CMP1 inhibits hTRPA1 through a noncovalent mechanism. In support of this, mTRPA1 and hTRPA1 responsiveness to AMG5445, a structural analog of CMP1, is essentially determined by the same residues as those for nonreactive menthol. This suggests that menthol and AMG5445 share a similar mechanism of action at TRPA1. These studies also raise the possibility that some reactive modulators of TRPA1 may simultaneously use both the covalent and noncovalent activation mechanisms.

In summary, we have demonstrated that TM5 determines the sensitivity of TRPA1 to various chemical modulators and identified specific residues governing species-dependent gating of TRPA1. These findings not only reveal a novel mechanism of TRPA1 modulation, but also have important implications for efforts to identify specific small molecule TRPA1 inhibitors.

\section{References}

Andersson DA, Gentry C, Moss S, Bevan S (2008) Transient receptor potential Al is a sensory receptor for multiple products of oxidative stress. J Neurosci 28:2485-2494.

Bandell M, Story GM, Hwang SW, Viswanath V, Eid SR, Petrus MJ, Earley TJ, Patapoutian A (2004) Noxious cold ion channel TRPA1 is activated by pungent compounds and bradykinin. Neuron 41:849-857.

Bandell M, Dubin AE, Petrus MJ, Orth A, Mathur J, Hwang SW, Patapoutian A 
(2006) High-throughput random mutagenesis screen reveals TRPM8 residues specifically required for activation by menthol. Nat Neurosci 9:493-500.

Bautista DM, Jordt SE, Nikai T, Tsuruda PR, Read AJ, Poblete J, Yamoah EN, Basbaum AI, Julius D (2006) TRPA1 mediates the inflammatory actions of environmental irritants and proalgesic agents. Cell 124:1269-1282.

Bautista DM, Siemens J, Glazer JM, Tsuruda PR, Basbaum AI, Stucky CL, Jordt SE, Julius D (2007) The menthol receptor TRPM8 is the principal detector of environmental cold. Nature 448:204-208.

Chen J, Zhang XF, Kort ME, Huth JR, Sun C, Miesbauer LJ, Cassar SC, Neelands T, Scott VE, Moreland RB, Reilly RM, Hajduk PJ, Kym PR, Hutchins CW, Faltynek CR (2008) Molecular determinants of species-specific activation or blockade of TRPA1 channels. J Neurosci 28:5063-5071.

Clapham DE (2003) TRP channels as cellular sensors. Nature 426:517-524.

Colburn RW, Lubin ML, Stone DJ Jr, Wang Y, Lawrence D, D’Andrea MR, Brandt MR, Liu Y, Flores CM, Qin N (2007) Attenuated cold sensitivity in TRPM8 null mice. Neuron 54:379-386.

Conaway CC, Krzeminski J, Amin S, Chung FL (2001) Decomposition rates of isothiocyanate conjugates determine their activity as inhibitors of cytochrome p450 enzymes. Chem Res Toxicol 14:1170-1176.

Dhaka A, Viswanath V, Patapoutian A (2006) Trp ion channels and temperature sensation. Annu Rev Neurosci 29:135-161.

Dhaka A, Murray AN, Mathur J, Earley TJ, Petrus MJ, Patapoutian A (2007) TRPM8 is required for cold sensation in mice. Neuron 54:371-378.

Doerner JF, Gisselmann G, Hatt H, Wetzel CH (2007) Transient receptor potential channel A1 is directly gated by calcium ions. J Biol Chem 282:13180-13189.

Doyle DA, Morais Cabral J, Pfuetzner RA, Kuo A, Gulbis JM, Cohen SL, Chait BT, MacKinnon R (1998) The structure of the potassium channel: molecular basis of $\mathrm{K}+$ conduction and selectivity. Science 280:69-77.

Eccles R (1994) Menthol and related cooling compounds. J Pharm Pharmacol 46:618-630.

Gavva NR, Tamir R, Klionsky L, Norman MH, Louis JC, Wild KD, Treanor JJ (2005) Proton activation does not alter antagonist interaction with the capsaicin-binding pocket of TRPV1. Mol Pharmacol 68:1524-1533.

Green BG (1992) The sensory effects of 1-menthol on human skin. Somatosens Mot Res 9:235-244.

Grimm C, Cuajungco MP, van Aken AF, Schnee M, Jörs S, Kros CJ, Ricci AJ, Heller S (2007) A helix-breaking mutation in TRPML3 leads to constitutive activity underlying deafness in the varitint-waddler mouse. Proc Natl Acad Sci USA 104:19583-19588.

Hill K, Schaefer M (2007) TRPA1 is differentially modulated by the amphipathic molecules trinitrophenol and chlorpromazine. J Biol Chem 282:7145-7153.

Hinman A, Chuang HH, Bautista DM, Julius D (2006) TRP channel activation by reversible covalent modification. Proc Natl Acad Sci U S A 103:19564-19568.

Jordt SE, Julius D (2002) Molecular basis for species-specific sensitivity to "hot" chili peppers. Cell 108:421-430.

Jordt SE, Bautista DM, Chuang HH, McKemy DD, Zygmunt PM, Högestätt ED, Meng ID, Julius D (2004) Mustard oils and cannabinoids excite sensory nerve fibres through the TRP channel ANKTM1. Nature 427:260-265.

Karashima Y, Damann N, Prenen J, Talavera K, Segal A, Voets T, Nilius B (2007) Bimodal action of menthol on the transient receptor potential channel TRPA1. J Neurosci 27:9874-9884.

Klionsky L, Tamir R, Gao B, Wang W, Immke DC, Nishimura N, Gavva NR (2007) Species-specific pharmacology of Trichloro(sulfanyl)ethyl benzamides as transient receptor potential ankyrin 1 (TRPA1) antagonists. Mol Pain 3:39.

Kwan KY, Allchorne AJ, Vollrath MA, Christensen AP, Zhang DS, Woolf CJ, Corey DP (2006) TRPA1 contributes to cold, mechanical, and chemical nociception but is not essential for hair-cell transduction. Neuron 50:277-289.

Lee SP, Buber MT, Yang Q, Cerne R, Cortés RY, Sprous DG, Bryant RW (2008) Thymol and related alkyl phenols activate the hTRPAl channel. Br J Pharmacol 153:1739-1749.

Levitt M (1978) Conformational preferences of amino acids in globular proteins. Biochemistry 17:4277-4285.

Levitt M, Greer J (1977) Automatic identification of secondary structure in globular proteins. J Mol Biol 114:181-239.

Long SB, Campbell EB, Mackinnon R (2005) Voltage sensor of Kv1.2: structural basis of electromechanical coupling. Science 309:903-908.

Macpherson LJ, Geierstanger BH, Viswanath V, Bandell M, Eid SR, Hwang S, Patapoutian A (2005) The pungency of garlic: activation of TRPA1 and TRPV1 in response to allicin. Curr Biol 15:929-934.
Macpherson LJ, Hwang SW, Miyamoto T, Dubin AE, Patapoutian A, Story GM (2006) More than cool: promiscuous relationships of menthol and other sensory compounds. Mol Cell Neurosci 32:335-343.

Macpherson LJ, Dubin AE, Evans MJ, Marr F, Schultz PG, Cravatt BF, Patapoutian A (2007a) Noxious compounds activate TRPA1 ion channels through covalent modification of cysteines. Nature 445:541-545.

Macpherson LJ, Xiao B, Kwan KY, Petrus MJ, Dubin AE, Hwang S, Cravatt B, Corey DP, Patapoutian A (2007b) An ion channel essential for sensing chemical damage. J Neurosci 27:11412-11415.

Maher M, Ao H, Banke T, Nasser N, Wu NT, Breitenbucher JG, Chaplan SR, Wickenden AD (2008) Activation of TRPA1 by farnesyl thiosalicylic acid. Mol Pharmacol 73:1225-1234.

McKemy DD, Neuhausser WM, Julius D (2002) Identification of a cold receptor reveals a general role for TRP channels in thermosensation. Nature 416:52-58.

McNamara CR, Mandel-Brehm J, Bautista DM, Siemens J, Deranian KL, Zhao M, Hayward NJ, Chong JA, Julius D, Moran MM, Fanger CM (2007) TRPAl mediates formalin-induced pain. Proc Natl Acad Sci U S A 104:13525-13530.

Meseguer V, Karashima Y, Talavera K, D’Hoedt D, Donovan-Rodríguez T, Viana F, Nilius B, Voets T (2008) Transient receptor potential channels in sensory neurons are targets of the antimycotic agent clotrimazole. J Neurosci 28:576-586.

Obata K, Katsura H, Mizushima T, Yamanaka H, Kobayashi K, Dai Y, Fukuoka T, Tokunaga A, Tominaga M, Noguchi K (2005) TRPA1 induced in sensory neurons contributes to cold hyperalgesia after inflammation and nerve injury. J Clin Invest 115:2393-2401.

Peier AM, Moqrich A, Hergarden AC, Reeve AJ, Andersson DA, Story GM, Earley TJ, Dragoni I, McIntyre P, Bevan S, Patapoutian A (2002) A TRP channel that senses cold stimuli and menthol. Cell 108:705-715.

Petrus M, Peier AM, Bandell M, Hwang SW, Huynh T, Olney N, Jegla T, Patapoutian A (2007) A role of TRPA1 in mechanical hyperalgesia is revealed by pharmacological inhibition. Mol Pain 3:40.

Phillips JC, Braun R, Wang W, Gumbart J, Tajkhorshid E, Villa E, Chipot C, Skeel RD, Kalé L, Schulten K (2005) Scalable molecular dynamics with NAMD. J Comput Chem 26:1781-1802.

Sanguinetti MC, Jiang C, Curran ME, Keating MT (1995) A mechanistic link between an inherited and an acquired cardiac arrhythmia: HERG encodes the IKr potassium channel. Cell 81:299-307.

Sawada Y, Hosokawa H, Hori A, Matsumura K, Kobayashi S (2007) Cold sensitivity of recombinant TRPA1 channels. Brain Res 1160:39-46.

Shelley JC, Cholleti A, Frye LL, Greenwood JR, Timlin MR, Uchimaya M (2007) Epik: a software program for $\mathrm{pK}(\mathrm{a})$ prediction and protonation state generation for drug-like molecules. J Comput Aided Mol Des 21:681-691.

Story GM, Peier AM, Reeve AJ, Eid SR, Mosbacher J, Hricik TR, Earley TJ, Hergarden AC, Andersson DA, Hwang SW, McIntyre P, Jegla T, Bevan S, Patapoutian A (2003) ANKTM1, a TRP-like channel expressed in nociceptive neurons, is activated by cold temperatures. Cell 112:819-829.

Trevisani M, Siemens J, Materazzi S, Bautista DM, Nassini R, Campi B, Imamachi N, Andrè E, Patacchini R, Cottrell GS, Gatti R, Basbaum AI, Bunnett NW, Julius D, Geppetti P (2007) 4-Hydroxynonenal, an endogenous aldehyde, causes pain and neurogenic inflammation through activation of the irritant receptor TRPA1. Proc Natl Acad Sci U S A 104:13519-13524.

Trudeau MC, Warmke JW, Ganetzky B, Robertson GA (1995) HERG, a human inward rectifier in the voltage-gated potassium channel family. Science 269:92-95.

Viswanath V, Story GM, Peier AM, Petrus MJ, Lee VM, Hwang SW, Patapoutian A, Jegla T (2003) Opposite thermosensor in fruitfly and mouse. Nature 423:822-823.

Voets T, Talavera K, Owsianik G, Nilius B (2005) Sensing with TRP channels. Nat Chem Biol 1:85-92.

Voets T, Owsianik G, Janssens A, Talavera K, Nilius B (2007) TRPM8 voltage sensor mutants reveal a mechanism for integrating thermal and chemical stimuli. Nat Chem Biol 3:174-182.

Vriens J, Janssens A, Prenen J, Nilius B, Wondergem R (2004) TRPV channels and modulation by hepatocyte growth factor/scatter factor in human hepatoblastoma (HepG2) cells. Cell Calcium 36:19-28.

Zhang XF, Chen J, Faltynek CR, Moreland RB, Neelands TR (2008) Transient receptor potential A1 mediates an osmotically activated ion channel. Eur J Neurosci 27:605-611.

Zurborg S, Yurgionas B, Jira JA, Caspani O, Heppenstall PA (2007) Direct activation of the ion channel TRPA1 by $\mathrm{Ca} 2+$. Nat Neurosci 10:277-279. 